\title{
Simply connected algebras of polynomial growth
}

\author{
ANDRZEJ SKOWROŃSKI \\ Faculty of Mathematics and Informatics, Nicholas Copernicus University, Chopina 12/18, \\ 87-100 Toruń, Poland.e-mail: skowron@mat.uni.torun.pl.
}

Received 7 November 1995; accepted in final form 26 August 1996

\begin{abstract}
The representation theory of polynomial growth strongly simply connected finite dimensional algebras over an algebraically closed field is established.
\end{abstract}

Mathematics Subject Classifications (1991): 16G20, 16G60, 16G70, 16 S90.

Key words: Simply connected, polynomial growth, Auslander-Reiten quiver

\section{Introduction}

Throughout by an algebra we mean a finite dimensional $K$-algebra with an identity over an algebraically closed field $K$. By a module over an algebra $A$ we mean a right $A$-module of finite $K$-dimension.

From Drozd's remarkable Tame and Wild Theorem [21] the class of algebras may be divided into two disjoint classes. One class consists of tame algebras for which the indecomposable modules occur, in each dimension $d$, in a finite number of discrete and a finite number of one-parameter families. The second class is formed by the wild algebras whose representation theory is as complicated as the study of finite dimensional vector spaces together with two non-commuting endomorphisms, for which the classification is a well-known unsolved problem. Hence, we can hope to classify the modules only for tame algebras. Frequently, applying covering techniques, we may reduce the study of modules over tame algebras to that for the corresponding simply connected algebras. Here, we are concerned with the representation theory of tame simply connected algebras.

Among the tame algebras we may distinguish the class of representation-finite algebras, having only finitely many isomorphism classes of indecomposable modules. This class of algebras is presently rather well understood (see [7], [13], [15], [16]). In particular, we know that if a representastion-finite algebra $A$ has a faithful indecomposable module $X$, then $A$ admits a Galois covering $\tilde{A}$ which has an indecomposable module $\tilde{X}$ whose push-down is $X$ and whose support is simply connected (see [14], [15], [16]). The class of sincere representation-finite simply connected algebras has been classified completely by quivers and relations. It consists of 24 infinite regular families of Bongartz [10] (whose Gabriel quiver has at least 14 vertices), being of considerable theoretical interest, and 16.344 exceptional 
algebras (see [18], [19], [40]). We note also that, for a representation-finite simply connected algebra $A$, the Auslander-Reiten quiver $\Gamma_{A}$ of $A$ is directed, and hence the indecomposable $A$-modules are uniquely determined by their dimension-vectors, being the positive roots of the Tits form $q_{A}$ of $A$ (see [11], [39]).

The representation theory of tame representation-infinite algebras is only emerging. At present the most accessible seem to be the (tame) algebras of polynomial growth, for which there exists an integer $m$ such that the number of one-parameter families is bounded, in each dimension $d$, by $d^{m}$. This class of algebras has been the subject of intensive research over the last 15 years. It contains the class of domestic algebras for which there is a constant bound on the number of one-parameter families in each dimension. Important classes of polynomial growth algebras are representation-infinite tilted algebras of Euclidean type and tubular algebras for which a complete representation theory was established in the Ringel's book [39]. Later O. Kerner proved in [24] that all tame tilted algebras are domestic, and hence of polynomial growth. Moreover, it was shown in [39] that the support of any indecomposable module which does not lie on a cycle of nonzero nonisomorphisms between indecomposable modules (directing module) is a tilted algebra. Hence, all tame algebras having sincere directing indecomposable modules are tilted and domestic. This class of tame algebras has been investigated by J. A. de la Peña in [33], [34]. On the other hand, by a result due to L. Peng-J. Xie [30] and the author [45], the Auslander-Reiten quiver of an algebra $A$ has at most finitely many $D T r$-orbits containing directing modules. Hence, in order to develop the representation theory of arbitrary tame algebras, we need informations on cycles of indecomposable modules. This was the main objective of studies in [3], [4], [5], [50] where a theory of algebras with finite cycles of indecomposable modules has been developed.

One of the main objectives of this paper is to describe the structure of the category mod $A$ of modules over an arbitrary strongly simply connected algebra $A$ of polynomial growth. Recall that an algebra $A$ without oriented cycles in its Gabriel quiver is called strongly simply connected [44] if, for any convex subcategory $C$ of $A$, the first Hochschild cohomology group $H^{1}(C, C)$ vanishes. For $A$ of finite representation type this coincides with the simply connectedness in the sense of [15] (the geometric realization $\left|\Gamma_{A}\right|$ of the Auslander-Reiten quiver $\Gamma_{A}$ of $A$ is simply connected). We characterize the polynomial growth of strongly simply connected algebras (Theorem 4.1) by properties of the infinite $\operatorname{radical} \operatorname{rad}^{\infty}(\bmod A)$ of $\bmod A$, the shape and behaviour of the Auslander-Reiten components in $\bmod A$, and properties of cycles of indecomposable modules in $\bmod A$. In particular, we get that, for a polynomial growth strongly simply connected $A$, all but finitely many $D$ Tr-orbits in $\Gamma_{A}$ are periodic, the components of $\Gamma_{A}$ are standard and partially ordered (in $\bmod A$ ), and each of them is either directed or a glueing of finitely many coils [4] by directed parts. Moreover, we obtain that a strongly simply connected algebra is of polynomial growth if and only if $A$ does not contain a convex subcategory which is hypercritical or $p g$-critical. This gives a handy criterion for 
the polynomial growth of strongly simply connected algebras. In the course of our proof of Theorem 4.1 (Section 6) we establish also the following important fact. For an arbitrary strongly simply connected algebra $A$ of polynomial growth, there are tame coil enlargements of critical algebras (coil algebras) [5] $B_{1}, \ldots, B_{n}$ which are convex subcategories of $A$ and whose indecomposable modules exhaust all but finitely many (up to isomorphism) indecomposable $A$-modules. Moreover, the remaining indecomposable modules are directing, and hence their supports are tame tilted algebras. This open a door to a complete classification of indecomposable modules over polynomial growth strongly simply connected algebras. We prove here (Corollary 4.7) that the one-parameter families of indecomposable modules over such algebras are given by the one-parameter families of indecomposable modules over critical and tubular algebras. In Section 4 we give also several characterizations of domestic strongly simply connected algebras (Corollary 4.3). In particular, we get that a strongly simply connected algebra $A$ is domestic if and only if $A$ does not contain a convex subcategory which is hypercritical, $p g$-critical or tubular.

We shall mention that our Theorem 4.1 is essentially applied in [52] to establish a handy criterion for the polynomial growth (respectively, domestic type) of algebras having strongly simply connected Galois coverings.

The results of this paper were partially announced in [46], [49], [51] and presented during the Conferences in Oberwolfach (1991), Ottawa (1992), Mexico (1994) and the Universities of Bielefeld, Paderborn, Trondheim, Paris VI, Nagoya, Tsukuba, Sherbrooke, Beijing, Singapore, Sao Paulo, Mexico. The author is grateful to these institutions for providing the possibility of discussing our investigations.

The research is also partially supported by Polish Scientific Grant KBN No. 2P03A 02008.

\section{Terminology and notation}

Throughout this paper $K$ will denote a fixed algebraically closed field. By an algebra is meant an associative finite dimensional $K$-algebra with an identity, which we shall assume (without loss of generality) to be basic. For such an algebra $A$, there exists an isomorphism $A \simeq K Q / I$, where $K Q$ is the path algebra of the Gabriel quiver $Q=Q_{A}$ of $A$ and $I$ is an admissible ideal in $K Q$. Equivalently, $A=K Q / I$ may be considered as $K$-category whose class of objects is the set $Q_{0}$ of vertices of $Q$, and the set of morphisms $A(x, y)$ from $x$ to $y$ is the quotient of the $K$-space $K Q(x, y)$, formed by the $K$-linear combinations of paths in $Q$ from $x$ to $y$, by the subspace $I(x, y)=K Q(x, y) \cap I$. An algebra $A$ with $Q_{A}$ having no oriented cycle is said to be triangular. A full subcategory $C$ of $A$ is said to be convex if any path in $Q_{A}$ with source and target in $Q_{C}$ lies entirely in $Q_{C}$.

For an algebra $A$, we denote by $\bmod A$ the category of finitely generated right $A$ modules and by ind $A$ its full subcategory consisting of indecomposable modules. The term $A$-module is used for an object of $\bmod A$ if not specified otherwise. 
We shall denote by $\operatorname{rad}(\bmod A)$ the Jacobson radical of $\bmod A$, that is, the ideal of $\bmod A$ generated by all noninvertible morphisms in ind $A$. Then the infinite radical $\operatorname{rad}^{\infty}(\bmod A)$ of $\bmod A$ is the intersection of all powers $\operatorname{rad}^{i}(\bmod A)$, $i \geqslant 1, \operatorname{of} \operatorname{rad}(\bmod A)$. We say that $\operatorname{rad}^{\infty}(\bmod A)$ is nilpotent (respectively, locally nilpotent) if there exists $m \geqslant 1$ such that $\left(\operatorname{rad}^{\infty}(\bmod A)\right)^{m}=0$ (respectively, $\left(\operatorname{rad}^{\infty}(M, M)\right)^{m}=0$ for any module $M$ in ind $A$ ). A path of length $t$ in $\bmod A$ is a sequence of nonzero nonisomorphisms

$$
M_{0} \stackrel{f_{1}}{\rightarrow} M_{1} \rightarrow \cdots \rightarrow M_{t-1} \stackrel{f_{t}}{\rightarrow} M_{t}
$$

with $t \geqslant 1$ and the modules $M_{i}$ in ind $A$. Such a path is said to be infinite if $f_{i} \in \operatorname{rad}^{\infty}(\bmod A)$ for some $1 \leqslant i \leqslant t$. A cycle of length $t$ is a path $(*)$ with $M_{0} \simeq M_{t}$. A module $M$ from ind $A$ is said to be directing if it does not lie on a cycle in $\bmod A$. For a module $M$ in $\bmod A$, we denote by $\operatorname{dim} M$ the dimensionvector $\left(\operatorname{dim}_{K} M(i)\right)_{i \in Q_{0}}$ of $M$. The support $\operatorname{supp} M$ of a module $M \in \bmod A$ is the full subcategory of $A$ given by all vertices $i \in Q_{A}$ such that $M(i) \neq 0$. We denote by $D: \bmod A \rightarrow \bmod A^{\text {op }}$ the standard duality $D=\operatorname{Hom}_{K}(-, K)$, where $A^{\text {op }}$ denotes the oposite algebra of $A$.

For an algebra $A$, we shall denote by $\Gamma_{A}$ the Auslander-Reiten quiver of $A$, and by $\tau_{A}$ and $\tau_{A}^{-}$the Auslander-Reiten translations $D \operatorname{Tr}$ and $\operatorname{Tr} D$, respectively. We shall identify the vertices of $\Gamma_{A}$ with the corresponding indecomposable $A$-modules. A connected component $\mathcal{C}$ of $\Gamma_{A}$ is said to be standard if the full subcategory of $\bmod A$ formed by all modules of $\mathcal{C}$ is equivalent to the mesh-category $K(\mathcal{C})$ of $\mathcal{C}$ (see [15], [39]). Moreover, following [47], a connected component $\mathcal{C}$ of $\Gamma_{A}$ is said to be generalized standard if $\operatorname{rad}^{\infty}(X, Y)=0$ for all modules $X$ and $Y$ in $\mathcal{C}$. It is known [28] that every standard component is generalized standard. For the components which do not contain both a projective module or an injective module, the converse implication is also true [48] but in general it is not the case. For a subquiver $\Gamma$ of $\Gamma_{A}$ we denote by $\operatorname{supp} \Gamma$ the support of $\Gamma$, that is, the full subcategory of $A$ formed by all vertices $x$ of $Q_{A}$ such that the simple $A$-modules $S(x)$ at $x$ occur as the composition factors of modules lying in $\Gamma$. Finally, the component quiver $\Sigma_{A}$ of $A$ [46] is defined as follows: the vertices of $\Sigma_{A}$ are the connected components of $\Gamma_{A}$, and two components $\mathcal{C}$ and $\mathcal{D}$ are connected in $\Sigma_{A}$ by an arrow $\mathcal{C} \rightarrow \mathcal{D}$ if and only if $\operatorname{rad}^{\infty}(X, Y) \neq 0$ for some modules $X$ in $\mathcal{C}$ and $Y$ in $\mathcal{D}$. Observe that a connected component $\mathcal{C}$ of $\Gamma_{A}$ is generalized standard if and only if $\Sigma_{A}$ has no loop at $\mathcal{C}$.

Let $A$ be an algebra and $K[x]$ the polynomial algebra in one variable. Following [21] $A$ is said to be tame if, for any dimension $d$, there exists a finite number of $K[x]-$ $A$-bimodules $M_{i}, 1 \leqslant i \leqslant n_{d}$, which are finitely generated and free as left $K[x]$ modules, and all but a finite number of isomorphism classes of indecomposable $A$-modules of dimension $d$ are of the form $K[x] /(x-\lambda) \otimes_{K[x]} M_{i}$ for some $\lambda \in K$ and some $i$. Let $\mu_{A}(d)$ be the least number of $K[x]-A$-bimodules satisfying the above conditions. Then $A$ is said to be of polynomial growth (respectively, of linear 
growth) if there exists a positive integer $m$ such that $\mu_{A}(d) \leqslant d^{m}$ (respectively, $\mu_{A}(d) \leqslant m d$ ) for any $d \geqslant 1$ [43]. Further, $A$ is domestic if $\mu_{A}(d) \leqslant m$ for a fixed $m \geqslant 1$ and any $d \geqslant 1$. From the validity of the second Brauer-Thrall conjecture we know that $A$ is representation-finite if and only if $\mu_{A}(d)=0$ for any $d \geqslant 1$. There is still an open problem whether all polynomial growth algebras are of linear growth.

We recall that, if $B$ is a representation-infinite tilted algebra of Euclidean type $\Delta$, then one of the following holds:

(a) $B$ is a domestic tubular extension of a tame concealed algebra $C$ and

$$
\Gamma_{B}=\mathcal{P}_{0} \vee \mathcal{T}_{0} \vee \mathcal{I}_{0},
$$

where $\mathcal{P}_{0}$ is the preprojective component of $\Gamma_{C}, \mathcal{T}_{0}$ is a $\mathbb{P}_{1}(K)$-family of pairwise orthogonal and standard ray tubes, obtained from the stable tubes of $\Gamma_{C}$ by ray insertions, and $\mathcal{I}_{0}$ a preinjective component containing a slice of type $\Delta$.

(b) $B$ is a domestic tubular coextension of a tame concealed algebra $C$ and

$$
\Gamma_{B}=\mathcal{P}_{\infty} \vee \mathcal{T}_{\infty} \vee \mathcal{I}_{\infty}
$$

where $\mathcal{P}_{\infty}$ is a preprojective component containing a slice of type $\Delta, \mathcal{T}_{\infty}$ is a $\mathbb{P}_{1}(K)$-family of pairwise orthogonal and standard coray tubes, obtained from the stable tubes of $\Gamma_{C}$ by coray insertions, and $\mathcal{I}_{\infty}$ is the preinjective component of $\Gamma_{C}$. The ordering from the left to the right indicates that there are nonzero morphisms only from any of these classes to itself and to the classes on its right (see [39, (4.9)]).

If $B$ is a tubular algebra then $B$ is a tubular extension (respectively, coextension) of a tame concealed algebra $C$ (respectively, $C^{\prime}$ ) and

$$
\Gamma_{B}=\mathcal{P}_{0} \vee \mathcal{T}_{0} \vee\left(\bigvee_{q \in \mathbb{Q}^{+}} \mathcal{T}_{q}\right) \vee \mathcal{T}_{\infty} \vee \mathcal{I}_{\infty}
$$

where $\mathcal{P}_{0}$ is the preprojective component of $\Gamma_{C}, \mathcal{T}_{0}$ is a $\mathbb{P}_{1}(K)$-family of pairwise orthogonal ray tubes, obtained from the stable tubes of $\Gamma_{C}$ by ray insertions, $\mathcal{I}_{\infty}$ is the preinjective component of $\Gamma_{C^{\prime}}, \mathcal{T}_{\infty}$ is a $\mathbb{P}_{1}(K)$-family of pairwise orthogonal coray tubes, obtained from the stable tubes of $\Gamma_{C^{\prime}}$ by coray insertions, and, for each $q \in \mathbb{Q}^{+}, \mathcal{T}_{q}$ is a $\mathbb{P}_{1}(K)$-family of pairwise orthogonal stable tubes. Moreover, all components of $\Gamma_{B}$ are standard (see [49, (5.2)]).

The Tits form of a triangular algebra $A=K Q / I$ is an integral quadratic form $q_{A}: \mathbb{Z}^{n} \rightarrow \mathbb{Z}, n=\left|Q_{0}\right|$, defined, for $x=\left(x_{i}\right) \in \mathbb{Z}^{n}$, by

$$
q_{A}(x)=\sum_{i \in Q_{0}} x_{i}^{2}-\sum_{(i \rightarrow j) \in Q_{1}} x_{i} x_{j}+\sum_{i, j \in Q_{0}} r(i, j) x_{i} x_{j}
$$


where $Q_{1}$ denotes the set of arrows in $Q$ and $r(i, j)$ is the cardinality of $L \cap$ $I(i, j)$, for a minimal set of generators $L \subset \bigcup_{i, j} I(i, j)$ of the ideal $I$. If moreover gl.dim $A \leqslant 2$, then $q_{A}$ coincides (see [11]) with the Euler form $\chi_{A}$ of $A$ which, for an $A$-module $X$, is defined by

$$
\chi_{A}(\underline{\operatorname{dim}} X)=\sum_{i=0}^{\infty}(-1)^{i} \operatorname{dim}_{K} \operatorname{Ext}_{A}^{i}(X, X),
$$

(see [39, (2.4)]). It is known (see [31]) that, if $A$ is tame, then $q_{A}$ is weakly nonnegative, that is, $q_{A}(x) \geqslant 0$ whenever $x \in \mathbb{N}^{n}$.

Finally, for an algebra $B$ and a $B$-module $R$ we denote by $B[R]$ the one-point extension algebra

$$
\left[\begin{array}{ll}
K & R \\
0 & B
\end{array}\right]
$$

For a basic background on the representation theory applied here we refer the reader to [22], [39], [4], [5].

\section{Simply connected algebras}

Let $A$ be a triangular algebra and $Q$ its Gabriel quiver. For each vertex $x$ of $Q$, denote by $Q(x)$ the subquiver of $Q$ obtained by deleting all those vertices of $Q$ being a source of a path in $Q$ with target $x$ (including the trivial path from $x$ to $x$ ). We shall denote by $A(x)$ the full subcategory of $A$ whose objects are the vertices of $Q(x)$. Moreover, for each vertex $x$ of $Q$, denote by $P(x)$ the indecomposable projective $A$-module at $x$, and by $R(x)$ the radical of $P(x)$. Then $R(x)$ is said to be separated if $R(x)$ is a direct sum of pairwise nonisomorphic indecomposable modules and whose supports are contained in pairwise different connected components of $Q(x)$. Following [8] we say that $A$ has the separation property if $R(x)$ is separated for any vertex $x$ of $Q$. It was shown in [44] that if $A$ has the separation property then $A$ is simply connected in the sense of [1], that is, for any presentation $A \simeq K Q / I$ of $A$ as a bound quiver algebra, the fundamental group $\pi_{1}(Q, I)$ of $(Q, I)$ is trivial. Recall also that $A$ is called strongly simply connected [44] if every convex subcategory of $A$ is simply connected. The following characterizations of strongly simply connected algebras have been proved in [44, (4.1)].

(2.1) PROPOSITION. For a triangular algebra $A$ the following conditions are equivalent:

(i) A is strongly simply connected.

(ii) Every convex subcategory of $A$ has the separation property.

(iii) Every convex subcategory of $A^{\mathrm{op}}$ has the separation property.

(iv) $H^{1}(C, C)=0$ for every convex subcategory $C$ of $A$. 
We note also that a representation-finite algebra $A$ is simply connected if and only if $A$ is strongly simply connected (see [16]). Moreover, the class of strongly simply connected algebras contains the class of completely separating algebras investigated by P. Dräxler [20].

We shall now exhibit some important classes of strongly simply connected algebras playing a crucial role in our investigations of polynomial growth algebras. Observe that a hereditary algebra is simply connected if and only if it is the path algebra of a tree. Let $\Delta$ be a finite connected quiver whose underlying graph $\bar{\Delta}$ is a tree, and $H=K \Delta$. Then it is known that $H$ is representation-infinite and tame if and only if $\bar{\Delta}$ is one of the Euclidean graphs
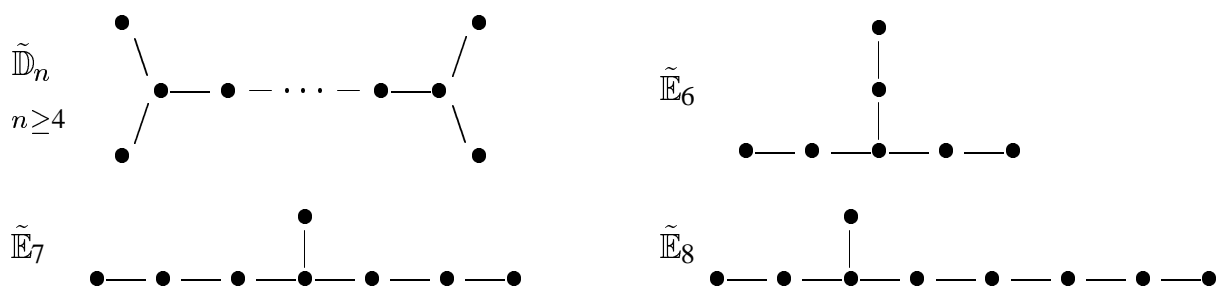

Hence $H=K \Delta$ is wild if and only if $\bar{\Delta}$ contains one of the following graphs

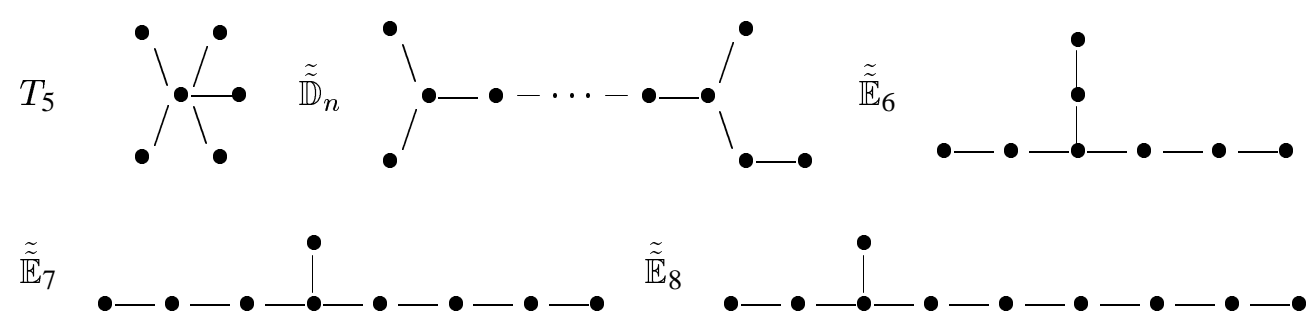

where in the case of $\tilde{\tilde{D}}_{n}$ the number of vertices is $n+2,4 \leqslant n \leqslant 8$.

Assume that $H=K \Delta$ is representation-infinite ( $\bar{\Delta}$ is not a Dynkin quiver) and $T$ is a preprojective tilting $H$-module, that is, $\operatorname{Ext}_{H}^{1}(T, T)=0$ and $T$ is a direct sum of $n=\left|\Delta_{0}\right|$ pairwise nonisomorphic indecomposable $H$-modules lying in the $\tau_{H}$-orbits of projective modules. Then $C=\operatorname{End}_{A}(T)$ is called a concealed algebra of type $\bar{\Delta}$. It is known that $\operatorname{gl} \operatorname{dim} C \leqslant 2$ and $C$ has the same representation type as $H$ (see [39]). A concealed algebra of type $\bar{\Delta}=\tilde{\mathbb{D}}_{n}, \tilde{\mathbb{E}}_{6}, \tilde{\mathbb{E}}_{7}$ or $\widetilde{\mathbb{E}}_{8}$ (respectively, $\bar{\Delta}=T_{5}, \tilde{\tilde{\mathbb{D}}}_{n}, \tilde{\mathbb{E}}_{6}, \tilde{\tilde{\mathbb{E}}}_{7}$ or $\tilde{\mathbb{E}}_{8}$ ) is said to be critical (respectively, hypercritical). The critical (respectively, hypercritical) algebras are strongly simply connected and have been classified completely by quivers and relations in [12], [23] (respectively, [26], [55], [56]). It is known (see [11], [12]) that a strongly simply connected algebra $A$ is representation-finite if and only if $A$ does not contain a critical convex subcategory, and if and only if $q_{A}$ is weakly positive (that is, $q_{A}(x)>0$ whenever $x \neq 0$ and has nonnegative coordinates). It is expected that a strongly simply connected algebra $A$ is tame if and only if $q_{A}$ is weakly nonnegative. We shall show in the paper that this is true for strongly simply connected algebras without 
$p g$-critical convex subcategories defined below. We shall use the following result proved in [32, (3.1)].

(2.2) PROPOSITION. Let $A$ be a strongly simply connected algebra. Then $q_{A}$ is weakly nonnegative if and only if $A$ does not contain a hypercritical convex subcategory.

For tubular extensions (respectively, coextensions) of critical algebras we have the following fact.

(2.3) PROPOSITION. Let B be a tubular extension (respectively, coextension) of a critical algebra $C$. Then the following conditions are equivalent:

(i) $B$ is tame.

(ii) $B$ is tubular or representation-infinite tilted of Euclidean type.

(iii) $q_{B}$ is weakly nonnegative.

Proof. Follows from [39] and [36, (3.3)].

Following [29] by a $p g$-critical algebra we mean an algebra $\Lambda$ satisfying the following conditions:

(i) $\Lambda$ is one of the matrix algebras

$$
B[R]=\left[\begin{array}{cc}
K & R \\
0 & B
\end{array}\right], \quad B[N, t]=\left[\begin{array}{ccccccc}
K & K & \ldots & K & K & K & N \\
& K & \ldots & K & K & K & 0 \\
& \ddots & \vdots & \vdots & \vdots & \vdots \\
& & & K & K & K & 0 \\
& & & & K & 0 & 0 \\
0 & & & & K & 0 \\
& & & & & & \\
& & & & & & B
\end{array}\right],
$$

where $B$ is a representation-infinite tilted algebra of Euclidean type $\tilde{\mathbb{D}}_{n}, n \geqslant 4$, with a complete slice in the preinjective component of $\Gamma_{B}, R$ (respectively, $N$ ) is an indecomposable regular $B$-module of regular length 2 (respectively, length 1) lying in a tube of $\Gamma_{B}$ with $n-2$ rays, $t+1(t \geqslant 2)$ is the number of isoclasses of simple $B[N, t]$-modules which are not $B$-modules.

(ii) Every proper convex subcategory of $\Lambda$ is of polynomial growth.

The $p g$-critical algebras have been classified by quivers and relations in [29]. In particular, it is shown in [29] that all $p g$-critical algebras are simply connected of global dimension 2 . There are 31 frames of such algebras, and among them only 16 frames are strongly simply connected.

The following proposition describes the representation type of hypercritical, $p g$-critical and tubular algebras. 
(2.4) PROPOSITION. (i) Every hypercritical algebra is wild.

(ii) Every pg-critical algebra is tame but not of polynomial growth.

(iii) Every tubular algebra is nondomestic of linear growth.

Proof. (i) It follows from [38] and [39, (4.2)], that, if $B$ is hypercritical, the $\Gamma_{B}$ has no homogeneous tube, and so $B$ is wild by [17, Corollary E]. See also [32, (3.1)], for a different proof.

(ii) For a proof we refer to [42].

(iii) See [43, Lemma 3.6].

(2.5) PROPOSITION. Let $B$ be a tubular algebra and

$$
\Gamma_{B}=\mathcal{P} \vee \mathcal{T}_{0} \vee\left(\bigvee_{q \in \mathbb{Q}^{+}} \mathcal{T}_{q}\right) \vee \mathcal{T}_{\infty} \vee \mathcal{I}_{\infty}
$$

the standard decomposition of $\Gamma_{B}$. Let $M$ be an indecomposable $B$-module lying in $\mathcal{P}_{0} \vee \mathcal{T}_{0} \vee\left(\bigvee_{q \in \mathbb{Q}^{+}} \mathcal{T}_{q}\right)$, and $\Lambda=B[M]$. Then $q_{\Lambda}$ is not weakly nonnegative.

Proof. Let $\omega$ be the extension vertex of $B[M]$. Since $M$ belongs to $\mathcal{P}_{0} \vee \mathcal{T}_{0} \vee$ $\left(\bigvee_{q \in \mathbb{Q}^{+}} \mathcal{T}_{q}\right)$ we have $\operatorname{pd}_{B} M \leqslant 1$ and hence gl.dim $\Lambda \leqslant 2$. In particular, $q_{\Lambda}=\chi_{\Lambda}$. Moreover, there exists $p \in \mathbb{Q}^{+}$such that $M$ belongs to $\mathcal{P}_{0} \vee \mathcal{T}_{0} \vee\left(\bigvee_{q<p} \mathcal{T}_{q}\right)$. Since $\mathcal{T}_{p}$ is separating (see [39, (3.1)]), there exist modules $X$ and $Y$ lying in different homogenous tubes of $\mathcal{T}_{p}$ such that $\operatorname{Hom}_{B}(M, X) \neq 0$ and $\operatorname{Hom}_{B}(M, Y) \neq 0$. Take $Z=X \oplus Y$ and put $v=\underline{\operatorname{dim}} Z+e_{\omega}$. Then

$$
\begin{aligned}
q_{\Lambda}(v)=\chi_{\Lambda}(v) & =\left\langle\underline{\operatorname{dim}} Z+e_{\omega}, \underline{\operatorname{dim}} Z+e_{\omega}\right\rangle \\
& =\langle\underline{\operatorname{dim}} Z, \underline{\operatorname{dim}} Z\rangle+\left\langle e_{\omega}, \underline{\operatorname{dim}} Z\right\rangle+\left\langle\underline{\operatorname{dim}} Z, e_{\omega}\right\rangle+\left\langle e_{\omega}, e_{\omega}\right\rangle \\
& =\left\langle e_{\omega}, \underline{\operatorname{dim}} Z\right\rangle+1=\langle\underline{\operatorname{dim}} P(\omega)-\underline{\operatorname{dim}} M, \underline{\operatorname{dim}} Z\rangle+1 \\
& =-\langle\underline{\operatorname{dim}} M, \underline{\operatorname{dim}} Z\rangle+1=-\operatorname{dim}_{K} \operatorname{Hom}_{B}(M, Z)+1<0,
\end{aligned}
$$

because $\operatorname{Ext}_{B}^{1}(M, Z) \simeq D \operatorname{Hom}_{B}\left(Z, \tau_{B} M\right)=0$. Therefore, $q_{\Lambda}$ is not weakly nonnegative.

(2.6) PROPOSITION. Let $B$ be a tubular extension of a critical algebra $C, \mathcal{T}$ a ray tube in $\Gamma_{B}$ obtained from a stable tube of $\Gamma_{C}$ by ray insertions, and $R$ an indecomposable $B$-module in $\mathcal{T}$ having two direct successors. Assume that $\Lambda=B[R]$ is strongly simply connected and does not contain a pg-critical convex subcategory. Then $q_{\Lambda}$ is not weakly nonnegative.

Proof. By Propositions 2.3 and 2.5, we may assume that $B$ is tilted of Euclidean type $\Delta\left(=\tilde{\mathbb{D}}_{n}, n \geqslant 4\right.$, or $\left.\tilde{\mathbb{E}}_{p}, 6 \leqslant p \leqslant 8\right)$. Let $r$ be the length of maximal sectional path in $\mathcal{T}$ with source $R$ and consisting of arrows pointing to the mouth. We know from [39, (4.9)] that $B=\operatorname{End}_{H}(T)$, where $H$ is a hereditary algebra of type $\Delta$ and $T$ a tilting $H$-module without preinjective direct summands. Moreover, $R=\operatorname{Hom}_{H}\left(T, R^{\prime}\right)$ for an indecomposable regular $H$-module $R^{\prime}$ of regular length $r$ lying in a stable tube $\mathcal{T}^{\prime}$ of $\Gamma_{H}$. Since by our assumption $\Lambda$ has no $p g$-critical 
convex subcategory, in the case $\Delta=\tilde{\mathbb{D}}_{n}$, we have additionally that $r \geqslant 3$ or $\mathcal{T}^{\prime}$ is not of rank $n-2$. Let $\omega$ be the extension vertex of $\Lambda=B[R]$. Then by [31, (2.1)], there are preinjective $H$-modules $V_{1}, \ldots, V_{m}$ and $a \in \mathbb{N}$ such that $q_{\Lambda}\left(\sum_{i=1}^{m} \operatorname{dim}_{H} \operatorname{Hom}_{H}\left(T, V_{i}\right)+a e_{\omega}\right)<0$, and hence $q_{\Lambda}$ is not weakly nonnegative.

(2.7) COROLLARY. Let $\Lambda$ be a strongly simply connected algebra of the form

$$
\Lambda=\left[\begin{array}{cc}
F & N^{r} \\
0 & C
\end{array}\right],
$$

where $C$ is a critical algebra, $N$ a simple regular $C$-module, $F$ is given by one of the quivers
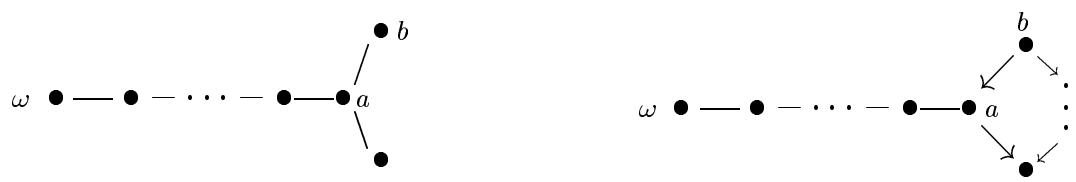

with the right quiver bound by the commutativity relation, and such that $\Lambda(x, y)=$ $N(y) \otimes_{K} F(x, \omega)$ for any vertices $x \in Q_{F}$ and $y \in Q_{C}$. Then either $\Lambda$ is pg-critical or $q_{\Lambda}$ is not weakly nonnegative.

Proof. Assume that $\Lambda$ is not $p g$-critical. In order to show that $q_{\Lambda}$ is not weakly nonnegative, we may assume that $a-b$ is oriented as $a \leftarrow b$. Let $B$ be the convex subcategory of $\Lambda$ given by all objects except $b$. Then $B$ is a tubular extension of $C$ and $\Lambda=B[R]$, where $R$ is the radical of $P(b)$. It is easy to see that $R$ is an indecomposable $B$-module lying in the ray tube $\mathcal{T}$ of $\Gamma_{B}$, obtained from the stable of $\Gamma_{C}$ containing $N$ by ray insertions, and has two direct successors in $\mathcal{T}$. Applying now the above proposition we infer that $q_{\Lambda}$ is not weakly nonnegative.

We shall need also the following fact.

(2.8) PROPOSITION. Let $A$ be a connected hereditary algebra of wild type, $T$ a tilting $H$-module without preprojective direct summands, and $B=\operatorname{End}_{H}(T)$. Then $q_{B}$ is not weakly nonnegative.

Proof. See [24, (6.2)].

\section{Infinite radical of module categories}

In this section we shall establish properties of the infinite radical of module categories over $p g$-critical, hypercritical and tubular algebras.

For a one-point extension $B[R]$ of an algebra $B$ by a $B$-module $R$, we identify $\bmod B[R]$ with the category of triples $(V, X, \varphi)$ where $V$ is a $K$-vector space, $X$ is a $B$-module and $\varphi: V \rightarrow \operatorname{Hom}_{B}(R, X)$ a $K$-linear map. Then a morphism $(V, X, \varphi) \rightarrow(W, Y, \psi)$ is given by a pair $(f, g)$ where $f: V \rightarrow W$ is a $K$ linear map, $g: X \rightarrow Y$ is a $B$-homomorphism and $\psi f=\operatorname{Hom}_{B}(R, g) \varphi$. For 
a background on the one-point extensions of algebras and vector space category methods we refer to [39] and [41].

We shall need the following lemma.

(3.1) LEMMA. Let $B$ be an algebra, $R$ a B-module, and $\varphi: Y \rightarrow Z a$ nonzero map in ind $B$. Assume that there are nonzero nonisomorphisms $f_{i}: W_{i} \rightarrow$ $W_{i+1}, i \geqslant 1$, in ind $B$ and nonzero maps $g: R \rightarrow Y, f_{0}: R \rightarrow W_{1}$ such that $\varphi g=0, f_{i} \ldots f_{0} \neq 0$ for $i \geqslant 1$, and $\operatorname{Hom}_{B}(R, h)=0$ for any $h: Y \rightarrow W_{i}$ or $h: W_{i} \rightarrow Y, i \geq 1$. Then $\varphi$ belongs to $\operatorname{rad}^{\infty}(\bmod B[R])$.

Proof. Since $_{\operatorname{Hom}}(R, h)=0$ for all maps $h: Y \rightarrow W_{i}$ or $h: W_{i} \rightarrow Y, i \geqslant 1$, we may consider the indecomposable $B[R]$-modules $Z_{i}=\left(K, Y \oplus W_{i}, \Delta_{i}\right), i \geqslant$ 1 , where $\Delta_{i}: K \rightarrow \operatorname{Hom}_{B}\left(R, Y \oplus W_{i}\right)=\operatorname{Hom}_{B}(R, Y) \oplus \operatorname{Hom}_{B}\left(R, W_{i}\right)$ is given by $\Delta_{i}(1)=\left(g, f_{i-1} \ldots f_{0}\right)$. Further, let $L$ be the indecomposable $B[R]$ module $(K, Y, \gamma)$ where $\gamma: K \rightarrow \operatorname{Hom}_{B}(R, Y)$ is given by $\gamma(1)=g$. For each $i \geqslant 1$, denote by $\alpha_{i}: Z_{i} \rightarrow Z_{i+1}$ the map $\left(1,\left(\begin{array}{cc}1 & 0 \\ 0 & f_{i}\end{array}\right)\right)$, and by $\beta_{i}: Z_{i} \rightarrow L$ the canonical map $(1,(1,0))$ induced by the projection $Y \oplus W_{i} \rightarrow Y$. Further, let $\alpha_{0}: Y=(0, Y, 0) \rightarrow\left(K, Y \oplus W_{1}, \Delta_{1}\right)=Z_{1}$ be the monomorphism given by the canonical map $Y \rightarrow Y \oplus W_{1}$. Finally, since $\varphi g=0$, we get also a nonzero map $\rho=(0, \varphi): L=(K, Y, \gamma) \rightarrow(0, Z, 0)=Z$. Observe that $\varphi=\rho \beta_{i+1} \alpha_{i} \ldots \alpha_{0}$ for any $i \geqslant 1$. Clearly, the maps $\alpha_{i}: Z_{i} \rightarrow Z_{i+1}$ are nonisomorphisms. Therefore, $\varphi \in \operatorname{rad}^{\infty}(\bmod B[R])$.

(3.2) PROPOSITION. Let A be a pg-critical algebra. Then $\bigcap_{m \geqslant 1}\left(\operatorname{rad}^{\infty}(\bmod A)\right)^{m}$ $\neq 0$ and $\operatorname{rad}^{\infty}(\bmod A)$ is not locally nilpotent.

Proof. Consider first the case when $A$ is of the form $B[N, t]$. Then $A$ is obtained from the one-point extension $B[N]$ by identifying its extension vertex with the vertex $\omega$ of the following quiver

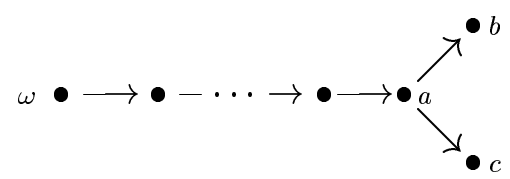

Denote by $B^{\prime}$ the convex subcategory of $A$ given by all objects of $A$ except $b$. Observe that $B^{\prime}$ is a representation-infinite tilted algebra of Euclidean type $\widetilde{\mathbb{D}}_{n+t}$ (if $B$ is of type $\tilde{\mathbb{D}_{n}}$ ) having a complete slice in the preinjective component of $\Gamma_{B^{\prime}}$ and $R^{\prime}=P_{B^{\prime}}(a)$ is an indecomposable regular $B^{\prime}$-module of regular length 2 lying in a ray tube of $\Gamma_{B^{\prime}}$ having $n+t-2$ rays. Hence $A^{\prime}=B^{\prime}\left[R^{\prime}\right]$ is a $p g$-critical algebra obtained from $B$ by reversing the arrow $a \rightarrow b$ on $a \leftarrow b$. Consider the APR-tilting module $T=\tau_{A}^{-}\left(S_{A}(b)\right) \oplus P$, where $P$ is given by $A=S_{A}(b) \oplus P$, associated to the simple $A$-module $S_{A}(b)$ at $b$. Then $A^{\prime} \simeq \operatorname{End}_{A}(T)$ and, by [6, Theorem 1.11], the functor $\operatorname{Hom}_{A}(T,-)$ induces an equivalence between the full subcategory of mod $A$ formed by all modules having no $S_{A}(b)$ as a direct summand and the full subcategory of mod $A^{\prime}$ formed by all modules having no $S_{A^{\prime}}(b)$ as a direct summand. Clearly, $\bigcap_{m \geqslant 1}\left(\operatorname{rad}^{\infty}(\bmod A)\right)^{m} \neq 0\left(\operatorname{respectively,} \operatorname{rad}^{\infty}(\bmod A)\right.$ 
is not locally nilpotent) if and only if $\bigcap_{m \geqslant 1}\left(\operatorname{rad}^{\infty}\left(\bmod A^{\prime}\right)\right)^{m} \neq 0$ (respectively, $\operatorname{rad}^{\infty}\left(\bmod A^{\prime}\right)$ is not locally nilpotent). Therefore, we may assume that $A$ is a one-point extension $A=B[R]$ where $B$ is a tilted algebra of type $\tilde{\mathbb{D}}_{s}$, being the corresponding tubular extension of the critical algebra $C$ of type $\tilde{\mathbb{D}}_{n}, R$ is an indecomposable $B$-module lying in a ray tube $\mathcal{C}$ of $\Gamma_{B}$ obtained from a stable tube $\mathcal{T}$ of rank $n-2$ of $\Gamma_{C}$ by a sequence of ray insertions, and such that we have in $\mathcal{C}$ a full translation subquiver of the form

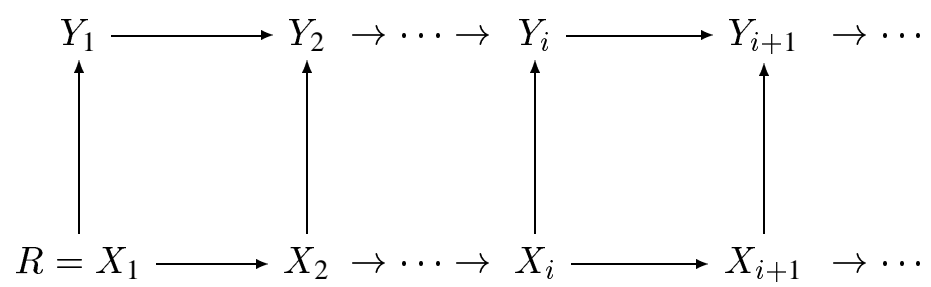

with $Y_{1}$ lying on the mouth of $\mathcal{C}$. Since $\mathcal{C}$ does not contain injective modules, the modules $Z_{i}=\tau_{B}^{-} Y_{i-1}, i \geqslant 2$, are nonzero. Fix $r \geqslant 2$ and take an arbitrary irreducible map $\varphi_{r}: Y_{r} \rightarrow Z_{r}$ in $\bmod B$. We claim that $\varphi_{r}$, considered as a map $\left(0, \varphi_{r}\right):\left(0, Y_{r}, 0\right) \rightarrow\left(0, Z_{r}, 0\right)$ in $\bmod B[R]=\bmod A$, belongs to $\operatorname{rad}^{\infty}(\bmod A)$. Choose arbitrary irreducible maps $u_{i}: X_{i} \rightarrow X_{i+1}, i \geqslant 1$, and $v: X_{r} \rightarrow Y_{r}$. Consider the modules $W_{i}=X_{r+i}, i \geqslant 1, Y=Y_{r}, Z=Z_{r}$ and the maps $\varphi=$ $\varphi_{r}: Y \rightarrow Z, g=v u_{r-1} \ldots u_{1}: R \rightarrow Y, f_{0}=u_{r} \ldots u_{1}: R \rightarrow W_{1}$, and $f_{i}=u_{r+i}: W_{i} \rightarrow W_{i+1}, i \geqslant 1$. Since $\mathcal{C}$ is a standard ray tube in $\Gamma_{B}$ and $Y_{1}$ lies on the mouth of $\mathcal{C}$ we get that $g \neq 0, \varphi g=0$ and $f_{i} \ldots f_{0} \neq 0$ for any $i \geq 1$. Moreover, $\operatorname{Hom}_{B}(R, h)=0$ for any $h: Y \rightarrow W_{i}$ or $h: W_{i} \rightarrow Y, i \geq 1$, in $\bmod B$. Hence, applying Lemma 3.1 we infer that $\varphi=\varphi_{r}$ belongs to $\operatorname{rad}^{\infty}(\bmod B[R])$.

We shall show now that $\bigcap_{m \geqslant 1}\left(\operatorname{rad}^{\infty}(\bmod A)\right)^{m} \neq 0$. Take an indecomposable $C$-module $U$ lying on the mouth of $\mathcal{T}$ and consider the sectional path

$$
\Sigma: \cdots \rightarrow U_{i} \rightarrow U_{i-1} \rightarrow \cdots \rightarrow U_{1} \rightarrow U_{0}=U
$$

in $\mathcal{T}$ consisting of arrows pointing to the mouth of $\mathcal{T}$. Let $P^{\prime}$ be an indecomposable projective $C$-module such that $\operatorname{Hom}_{C}\left(P^{\prime}, U\right) \neq 0$. Let $f: P^{\prime} \rightarrow U$ be a nonzero map. Clearly, $f \in \operatorname{rad}^{\infty}\left(P^{\prime}, U\right)$ because $P^{\prime}$ is not in $\mathcal{T}$. Since $\mathcal{T}$ is a standard stable tube in $\Gamma_{C}$, there are irreducible morphisms $g_{i}: U_{i} \rightarrow U_{i-1}$ and morphisms $h_{i}: P^{\prime} \rightarrow U_{i}, i \geqslant 1$, in $\bmod C$ such that $f=g_{1} \ldots g_{i} h_{i}$ for any $i \geqslant 1$. The tube $\mathcal{C}$ of $\Gamma_{B}$ is obtained from $\mathcal{T}$ by a sequence of ray insertions, and hence each arrow $U_{i} \rightarrow U_{i-1}$ of $\mathcal{T}$ either remains an arrow of $\mathcal{C}$ or is replaced by a finite sectional path $U_{i} \rightarrow \cdots \rightarrow U_{i-1}$ in $\mathcal{C}$, consisting of modules pointing to the mouth of $\mathcal{C}$. Hence the modules $U_{i}, i \geqslant 0$, lie on a sectional path $\Sigma^{\prime}$ of $\mathcal{C}$ consisting of modules pointing to the mouth. Observe that the intersection of $\Sigma^{\prime}$ with the sectional path

$$
\Omega: Y_{1} \rightarrow Y_{2} \rightarrow \cdots \rightarrow Y_{j} \rightarrow Y_{j+1} \rightarrow \cdots,
$$


is infinite. Therefore, there exists a sequence $1 \leqslant i_{1}<i_{2}<i_{3}<\ldots$ of positive integers such that, for each $s \geqslant 1$, the subpath of $\Sigma^{\prime}$ with source $U_{i_{s}}$ and target $U_{i_{s-1}}$ contains a module, say $Y_{j_{s}}$, of $\Omega$, different from $U_{i_{s-1}}$. Then $g_{i_{s}}=\eta_{s} \psi_{s} \xi_{s}$ for an irreducible map $\psi_{s}: Y_{j_{s}} \rightarrow Z_{j_{s}}$ and some maps $\xi_{s}$ : $U_{i_{s}} \rightarrow Y_{j_{s}}$ and $\eta_{s}: Z_{j_{s}} \rightarrow U_{i_{s-1}}$ in $\bmod B$. We know that the maps $\psi_{s}$ belong to $\operatorname{rad}^{\infty}(\bmod B[R])=\operatorname{rad}^{\infty}(\bmod A)$, and so $g_{i_{s}}$ also belong to $\operatorname{rad}^{\infty}(\bmod A)$. Consequently, $f \in \bigcap_{m \geqslant 1}\left(\operatorname{rad}^{\infty}(\bmod A)\right)^{m}$. This proves that $\bigcap_{m \geqslant 1}\left(\operatorname{rad}^{\infty}(\bmod A)\right)^{m} \neq$ 0 . Finally, we prove that $\operatorname{rad}^{\infty}(\bmod A)$ is not locally nilpotent. Fix $m \geqslant 1$. Take an indecomposable $C$-module $X$ in $\mathcal{T}$ of quasi-length $(m+1)(n-1)$, where $n-2$ is the rank of $\mathcal{T}$. Since the full subcategory of $\bmod C$ given by the modules in the standard stable tube $\mathcal{T}$ is serial, we infer that $\operatorname{End}_{C}(X) \simeq K[T] /\left(T^{m+1}\right)$ (see [39, (3.1)]). Consider a path of irreducible epimorphisms

$$
X=X_{0} \stackrel{u_{1}}{\longrightarrow} X_{1} \longrightarrow \cdots \stackrel{u_{n-2}}{\longrightarrow} X_{n-2}=W
$$

and a path of irreducible monomorphisms

$$
W=Y_{n-2} \stackrel{v_{n-2}}{\longrightarrow} \cdots \longrightarrow Y_{1} \stackrel{v_{1}}{\longrightarrow} Y_{0}=X,
$$

in $\bmod C$, and put $v=v_{1} \ldots v_{n-2}, u=u_{n-2} \ldots u_{1}, h=v u$. Then $h \in \operatorname{rad}(X, X)$ and $h^{m} \neq 0$. As above we conclude that in $\bmod B$ the morphism $u$ has a decomposition $u=\eta \psi \xi$ for an irreducible map $\psi: Y_{i} \rightarrow Z_{i}$ and some maps $\xi: X \rightarrow Y_{i}$ and $\eta: Z_{i} \rightarrow X_{n-2}$. Since $\psi$ belongs to $\operatorname{rad}^{\infty}(\bmod A)$, we get that $h=v u$ also belongs to $\operatorname{rad}^{\infty}(\bmod A)$. Therefore, $\left(\operatorname{rad}^{\infty}(X, X)\right)^{m} \neq 0$. This proves that $\operatorname{rad}^{\infty}(\bmod A)$ is not locally nilpotent.

(3.3) PROPOSITION. Let $A$ be an algebra such that $\bigcap_{m \geqslant 1}\left(\operatorname{rad}^{\infty}(\bmod A)\right)^{m}=0$ or $\operatorname{rad}^{\infty}(\bmod A)$ is locally nilpotent. Then $A$ is tame.

Proof. Suppose that $A$ is not tame. Then By [21] (see also [17]), $A$ is wild, that is, denoting by $K\langle x, y\rangle$ the free $K$-algebra in two noncommuting variables $x$ and $y$, there exists a $K\langle x, y\rangle-A$-bimodule $M$, free and finitely generated as a left $K\langle x, y\rangle$-module, such that the functor $F:-\otimes_{K\langle x, y\rangle} M: \bmod K\langle x, y\rangle \rightarrow \bmod A$ preserves indecomposability and isomorphism classes; in particular $F$ is faithful. Let now $\Lambda$ be a $p g$-critical algebra. It is well-known that there exists a full exact embedding $G: \bmod \Lambda \rightarrow \bmod K\langle x, y\rangle$. Hence, we get a faithful functor $F G: \bmod \Lambda \rightarrow \bmod A$. From Proposition 3.2 we infer that there is a nonzero morphism $f$ in $\bigcap_{m \geqslant 1} \operatorname{rad}^{\infty}(\bmod \Lambda)^{m}$. Then $F G(f) \neq 0$ and belongs to $\bigcap_{m \geqslant 1}\left(\operatorname{rad}^{\infty}(\bmod A)\right)^{m}$. Similarly, for each $m \geq 1$, there exists a module $X \in$ ind $\Lambda$ and a nonzero morphism $h_{m}$ in $\left(\operatorname{rad}^{\infty}(X, X)\right)^{m}$. Then $F G\left(h_{m}\right) \neq 0$ and belongs $\left(\operatorname{rad}^{\infty}(F G(X), F G(X))\right)^{m}$. Therefore, we get that $\bigcap_{m>1}\left(\operatorname{rad}^{\infty}(\bmod A)\right)^{m}$ $\neq 0$ and $\operatorname{rad}^{\infty}(\bmod A)$ is not locally nilpotent, a contradiction. This proves that $A$ is tame.

As a direct consequence of Propositions 2.4 and 3.3 we get the following fact.

(3.4) COROLLARY. Let A be a hypercritical algebra. Then $\bigcap_{m \geqslant 1}\left(\operatorname{rad}^{\infty}(\bmod A)\right)^{m}$ $\neq 0$ and $\operatorname{rad}^{\infty}(\bmod A)$ is not locally nilpotent. 
We shall need also the following fact.

(3.5) PROPOSITION. Let $A$ be a tubular algebra. Then

$$
\operatorname{rad}^{\infty}(A, D(A))=\left(\operatorname{rad}^{\infty}(A, D(A))\right)^{2}=\left(\operatorname{rad}^{\infty}(A, D(A))^{3}=\ldots\right.
$$

In particular, $\bigcap_{m \geqslant 1}\left(\operatorname{rad}^{\infty}(\bmod A)\right)^{m} \neq 0$.

Proof. It is a direct consequence of the separation property of tubular families in $\Gamma_{A}[39,(5.2)]$. For a complete proof we refer to $[25,(1.5)]$.

\section{Strongly simply connected algebras of polynomial growth}

The following theorem gives different characterizations of polynomial growth strongly simply connected algebras and their module categories.

(4.1) THEOREM. Let A be a strongly simply connected algebra. The following conditions are equivalent:

(i) A is of polynomial growth.

(ii) $A$ is of linear growth.

(iii) A does not contain a convex subcategory which is pg-critical or hypercritical.

(iv) $\operatorname{rad}^{\infty}(\bmod A)$ is locally nilpotent.

(v) $\Sigma_{A}$ is directed.

(vi) Every connected component of $\Gamma_{A}$ is standard.

(vii) $A$ is a multicoil algebra.

The proof will be presented in Section 6. Here, we shall derive several important consequences of the above theorem. First observe that the condition (iii) gives a handy criterion for a strongly simply connected algebra to be of polynomial (respectively, linear) growth. The condition (v) gives a partial order of all connected component of $\Gamma_{A}$ in $\bmod A$. In particular, it implies that every cycle in $\bmod A$ is finite ( $A$ is cycle-finite in the sense of [2]), and so $\operatorname{rad}^{\infty}(M, M)=0$ for any module $M$ from ind $A$. Moreover, the condition (vii) gives a more precise description of cycles in $\bmod A$. Namely, there exists a natural generalization of the notion of a tube called a coil (see [3], [4], [5]). Then a multicoil consists, roughly speaking, of a finite number of coils glued together by some directed parts, and a multicoil algebra is an algebra $A$ having the property that every cycle in $\bmod A$ consists of modules of a (standard) coil of a multicoil of $\Gamma_{A}$.

The following corollary is a direct consequence of Theorem 4.1, Proposition 2.2 and the fact that the hypercritical algebras are wild.

(4.2) COROLLARY. Let $A$ be a strongly simply connected algebra having no pg-critical convex subcategory. The following conditions are equivalent:

(i) $A$ is tame.

(ii) $A$ is of polynomial growth.

(iii) $q_{A}$ is weakly nonnegative. 
The following corollary gives characterizations of domestic strongly simply connected algebras and their module categories.

(4.3) COROLLARY. Let A be a strongly simply connected algebra. The following conditions are equivalent:

(i) $A$ is domestic.

(ii) A does not contain a convex subcategory which is hypercritical, pg-critical or tubular.

(iii) $\bigcap_{m \geqslant 1}\left(\operatorname{rad}^{\infty}(\bmod A)\right)^{m}=0$.

(iv) $\operatorname{rad}^{\infty}(\bmod A)$ is nilpotent.

(v) $\Sigma_{A}$ is directed and there is a common bound on the length of paths in $\Sigma_{A}$.

Proof. It follows from Theorem 4.1 that, if $A$ is of polynomial growth, then every cycle in $\bmod A$ is finite. Then the equivalence of (i) and (ii) is a direct consequence of Theorem 5.1 in [50]. Clearly, (v) implies (iv), and (iv) implies (iii). Moreover, by Propositions 3.2, 3.5 and Corollary 3.4, (iii) implies (ii). Therefore, it remains to show that (ii) implies (v). Assume that (ii) holds. Then, by Theorem 4.1, $\Sigma_{A}$ is directed and every connected component of $\Gamma_{A}$ is (generalized) standard. Applying now [47, Corollary 3.11], we infer that all but finitely many connected components of $\Gamma_{A}$ are stable tubes. Further, since $A$ is a cycle-finite algebra, we infer by Theorem 4.1 in [50] that the support supp $\mathcal{T}$ of any stable tube $\mathcal{T}$ in $\Gamma_{A}$ is a convex critical or tubular subcategory of $A$. Moreover, the stable tubes in the Auslander-Reiten quiver of any critical algebra are pairwise orthogonal and standard. Therefore, the fact that $A$ has no tubular convex subcategory, implies that the paths in $\Sigma_{A}$ are of bounded length. This finishes the proof.

Recall that an Auslander-Reiten quiver $\Gamma_{A}$ is called quasi-periodic if all but finitely many $\tau_{A}$-orbits in $\Gamma_{A}$ are periodic.

(4.4) COROLLARY. Let A be a strongly simply connected algebra of polynomial growth. Then $\Gamma_{A}$ is quasi-periodic and all but finitely many components in $\Gamma_{A}$ are stable tubes. If $A$ is domestic then all but finitelly many components in $\Gamma_{A}$ are stable tubes of rank 1.

Proof. From [47, Theorem 2.3], every generalized standard component of $\Gamma_{A}$ admits at most finitely many nonperiodic $\tau_{A}$-orbits. Further, since $A$ is cyclefinite, we know by [50, Proposition 3.3], that every regular generalized standard component of $\Gamma_{A}$ is a stable tube. Hence our claim follows from Theorem 4.1(vi) and [50, Theorem 4.4].

For a component $\mathcal{C}$ of $\Gamma_{A}$ we denote by $|\mathcal{C}|$ the geometric realization of $\mathcal{C}$ as defined in [15, (4.1)]. Moreover, following [4, (3.3)], a coil $\Gamma$ in $\Gamma_{A}$ is said to be proper if each of its vertices belongs to an oriented cycle of $\Gamma$. As a direct consequence of our proof of Theorem 4.1 given in Section 6 we get the following fact. 
(4.5) COROLLARY. Let A be a strongly simply connected algebra of polynomial growth, $\mathcal{C}$ a (connected) component of $\Gamma_{A}, X$ a vertex of $\Gamma_{A}$, and $r$ the number of pairwise disjoint proper coils in $\mathcal{C}$. Then the fundamental group $\pi_{1}(|\mathcal{C}|, X)$ is a free (noncommutative) group in $r$ generators.

We shall now derive some informations on the supports of indecomposable modules over strongly simply connected algebras of polynomial growth.

(4.6) COROLLARY. Let A be a strongly simply connected algebra of polynomial growth and $\mathcal{T}$ a stable tube in $\Gamma_{A}$. Then the support of $\mathcal{T}$ is a critical or tubular convex subcategory of $A$.

Proof. See [3, (4.1)], or [50, (4.2)].

The following corollary describes the one-parameter families of indecomposable modules over strongly simply connected algebras of polynomial growth.

(4.7) COROLLARY. Let A be a strongly simply connected algebra of polynomial growth. Assume that $M$ is a module in ind $A$ such that there are infinitely many pairwise nonisomorphic modules $N$ in ind $A$ with $\underline{\operatorname{dim}} N=\underline{\operatorname{dim}} M$. Then $\operatorname{supp} M$ is a convex critical or tubular subcategory of $A$.

Proof. Since $A$ is tame, we know from [17] that all but finitely many isoclasses of indecomposable $A$-modules of dimension-vector $\underline{\operatorname{dim}} M$ lie in the stable tubes of rank 1 . Hence, by our assumption, $\underline{\operatorname{dim}} M=\underline{\operatorname{dim}} N$ for a module $N$ lying in a stable tube $\mathcal{T}$ of rank 1. Consequently, by the above corollary, $\operatorname{supp} M=\operatorname{supp} N=$ supp $\mathcal{T}$ is a convex critical or tubular subcategory of $A$.

By a coil algebra we mean a tame coil enlargement of a tame concealed algebra (in the sense of [5]). As a direct consequence of the proof of Theorem 4.1 presented in Section 6 we get also the following fact.

(4.8) COROLLARY. Let A be a strongly simply connected algebra of polynomial growth. Then there exist convex coil subcategories $B_{1}, \ldots B_{m}$ of $A$ whose indecomposable modules exhaust all but finitely many isoclasses of indecomposable $A$-modules. Moreover, if the support $D=\operatorname{supp} X$ of an indecomposable A-module $X$ is not contained in one of the categories $B_{1}, \ldots, B_{m}$, then $X$ is directing, and so $D$ is a tame tilted algebra.

We shall note that the module categories over coil algebras are well understood (see [5]). Moreover, a complete classification of coil algebras with sincere nondirecting indecomposable modules lying in nonstable coils will be presented in a joint paper with I. Assem and B. Tomé. Hence, in view of Corollary 4.5, in order to classify the indecomposable modules over strongly simply connected algebras of polynomial growth, it remains to describe the supports of directing modules. If $X$ is a directing module over such an algebra $A$ then $\operatorname{supp} X$ is a convex subcategory of $A$ [11, Proposition 3.2], and is tilted [39, p. 376]. Hence, we need a classification of tame tilted strongly simply connected algebras with sincere directing modules. Those of finite representation type are classified in [10] and [18], [40]. It was shown 
in [33] that the representation-infinite tame tilted algebras with sincere directing modules have, in each dimension $d$, at most 2 one-parameter families of modules. The families of two-parametric tame algebras with sincere directing modules and having at least 20 vertices in the Gabriel quiver have been classified in [34]. The classification of the remaining ones is still an open problem.

The support of a nondirecting indecomposable module over a polynomial growth strongly simply connected algebra $A$ is not necessarily a convex subcategory of $A$. The following simple example is due to P. Dräxler. Namely, let $C$ be the critical algebra of type $\tilde{\mathbb{D}}_{5}$ given by the fully commutative quiver

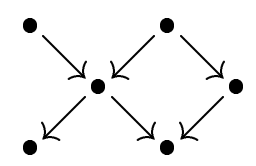

and $M$ be the indecomposable $C$-module

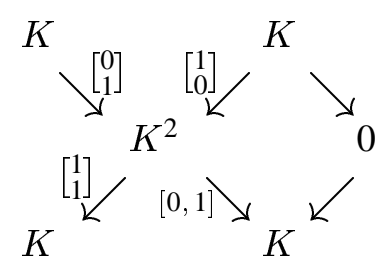

Then clearly supp $M$ is not a convex subcategory of $C$. But from the above discussion and our proof of Theorem 4.1 we can deduce the following fact.

(4.9) COROLLARY. Let A be a strongly simply connected algebra of polynomial growth, $M$ a module in ind $A$ and $\Lambda=\operatorname{supp} M$.

(i) If $\Lambda$ is representation-finite then $\Lambda$ is a convex subcategory of $A$.

(ii) If $\Lambda$ is representation-infinite, then it contains a full subcategory $C$ which is critical.

For $M \in$ ind $A$, denote by $c(M)$ the number of pairwise different critical convex subcategories of the convex hull of supp $M$ in $A$. Then we have the following fact.

(4.10) COROLLARY. Let A be a strongly simply connected algebra of polynomial growth and $M$ a module in ind $A$. Then $c(M) \leqslant 3$. Moreover, if $A$ is domestic, then $c(M) \leqslant 2$.

Proof. It is a direct consequence of Corollaries 4.7 and 4.8, [4], [5, (4.2)], and [33].

In the joint work with $\mathrm{J}$. A. de la Peña [35] we proved that, if $A$ is a sincere tame strongly simply connected algebra and admits a convex subcategory which is either tubular or representation-infinite tilted of type $\tilde{E}_{p}, 6 \leqslant p \leqslant 8$, then $A$ is of polynomial growth and its quiver $Q_{A}$ has at most 19 vertices. Then it follows (see [35, Corollary 6.2]) that every sincere polynomial growth strongly simply connected algebra with at least 14 vertices is domestic. 
We end this section with an example illustrating our criteria for the polynomial growth and domestic type of strongly simply connected algebras.

Let $A=K Q / I$ where $Q$ is the quiver

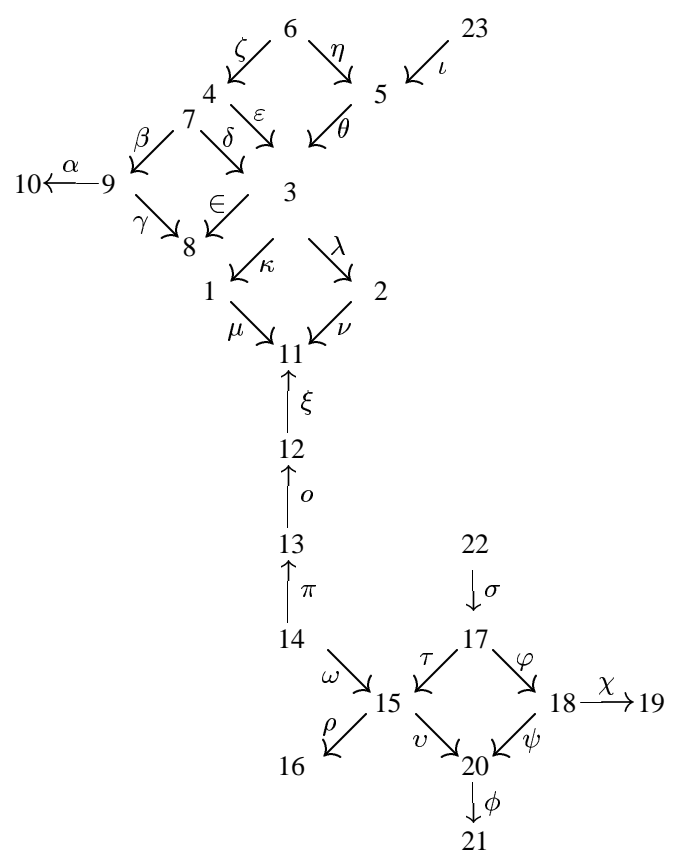

and $I$ is the ideal of $K Q$ generated by $\beta \gamma-\delta \in, \zeta \varepsilon-\eta \theta, \kappa \mu-\lambda \nu, \delta \kappa, \delta \lambda, \varepsilon \in, \theta \in$, $\iota \theta \lambda, \pi o \xi, \omega \rho, \omega v, \tau v-\varphi \psi$. Clearly, $A$ is strongly simply connected. A simple inspection of the frames of critical algebras presented in [12] and [23] shows that the following algebras form a complete set of critical convex subcategories of $A$ :

$C_{1}$ (of type $\tilde{\mathbb{D}}_{4}$ ) given by the vertices $1,2,3,4,5$;

$C_{2}$ (of type $\tilde{\mathbb{E}}_{8}$ ) given by the vertices $1,3,4,5,6,7,9,10,23$;

$C_{3}$ (of type $\tilde{\mathbb{E}}_{6}$ ) given by the vertices $15,16,17,18,19,20,21$;

$C_{4}$ (of type $\tilde{\mathbb{E}}_{6}$ ) given by the vertices $15,16,17,18,19,20,22$;

$C_{5}$ (of type $\tilde{\mathbb{E}}_{8}$ ) given by the vertices $12,13,14,15,16,17,18,19,22$.

Similarly, a simple inspection of the frames of hypercritical algebras presented in [55] shows that $A$ does not contain a convex subcategory which is hypercritical. Further, there is no extension of $C_{1}$ to a $p g$-critical convex subcategory of $A$, and hence $A$ does not contain a convex subcategory which is $p g$-critical. Hence, applying Theorem 4.1, we conclude that $A$ is of polynomial growth. Further, it is easy to see that the following algebras form a complete set of tubular convex subcategories of $A$ :

$\Lambda_{1}$ (of type $(2,3,6)$ ) given by the vertices $1,2,3,4,5,6,7,9,10,23$;

$\Lambda_{2}$ (of type $(2,3,6)$ ) given by the vertices $12,13,14,15,16,17,18,19,20,22$;

$\Lambda_{3}$ (of type $(3,3,3)$ ) given by the vertices $15,16,17,18,19,20,21,22$. 
In particular, by Corollary 4.3, $A$ is not domestic. On the other hand, the full convex subcategory $A^{\prime}$ of $A$ formed by all objects of $A$ except 22 and 23 is a representationinfinite domestic strongly simply connected algebra, containing only two critical convex subcategories, namely $C_{1}$ and $C_{3}$. Finally, we note that the algebras:

$B_{1}$ given by the vertices $1,2,3, \ldots, 12,13,23$;

and

$B_{2}$ given by the vertices $12,13,14, \ldots, 20,21,22$,

are convex coil subcategories of $A$ whose indecomposable modules exhaust (up to isomorphism) all indecomposable $A$-modules.

\section{Partial order of sources}

Let $A=K Q / I$ be an algebra with the separation property and $\mathcal{S}$ the set of all sources in $Q$. The aim of this section is to establish a partial order $\leqslant$ in $\mathcal{S}$ in the case when all component quivers $\Sigma_{A(x)}, x \in \mathcal{S}$, are directed. This will play a crucial role in our proof of Theorem 4.1. We start with the following technical lemma.

(5.1) LEMMA. Let $z \in \mathcal{S}$ and $\mathcal{C}$ be a connected component of $\Gamma_{A(z)}$. Assume that $\Sigma_{A(z)}$ is directed and there exists an infinite path

$$
Z=Z_{0} \rightarrow Z_{1} \rightarrow \cdots \rightarrow Z_{s}=N,
$$

in $\bmod A(z)$ such that $Z$ belongs to $\mathcal{C}$ and $N$ is an indecomposable direct summand of $R(z)$. Moreover, let

$$
\mathcal{D}=\mathcal{C}_{0} \rightarrow \cdots \rightarrow \mathcal{C}_{t}=\mathcal{C},
$$

$t \geqslant 0$, be a path in $\Sigma_{A(z)}$ and $Y$ a module in $\mathcal{D}$. Then every indecomposable $A$ module $M$ lying on a path in $\bmod A$ with target $Y$ is an $A(z)$-module. In particular, $\mathcal{D}$ is a full component of $\Gamma_{A}$.

Proof. Let $M=M_{0} \rightarrow M_{1} \rightarrow \cdots \rightarrow M_{r}=Y$ be a path in $\bmod A$ with target $Y$. Suppose that $\operatorname{Hom}_{A}(P(z), M) \neq 0$. We may assume that $M_{1}, \ldots, M_{r}$ are $A(z)$ modules. We shall show that $\Sigma_{A(z)}$ admits an oriented cycle $\mathcal{C} \rightarrow \cdots \rightarrow \mathcal{D} \rightarrow$ $\cdots \rightarrow \mathcal{C}$, which will contradict our assumption on $\Sigma_{A(z)}$. Since $A=A(z)[R(z)]$ we may consider $M$ as a triple $(V, X, \varphi)$ where $V$ is a $K$-vector space, $X$ an $A(z)$ module, and $\varphi: V \rightarrow \operatorname{Hom}_{A(z)}(R(z), X)$ a $K$-linear map. Observe that $M$ is not isomorphic to the simple injective $A$-module $S(z)=(K, 0,0)$, because there is a nonzero nonisomorphism $M \rightarrow M_{1}$ with $M_{1}$ indecomposable. Hence $X \neq 0$ and let $X=X_{1} \oplus \ldots \oplus X_{r}$ be a decomposition of $X$ into a direct sum of indecomposable $A(z)$-modules. Since $\operatorname{Hom}_{A}(P(z), M) \neq 0$ and $M$ is indecomposable, we infer that $\varphi \neq 0$ and $\operatorname{Hom}_{A(z)}\left(R(z), X_{i}\right) \neq 0$ for any $1 \leq i \leq r$. Moreover, since $\operatorname{Hom}_{A}\left(M, M_{1}\right) \neq 0$ and $M_{1}=\left(0, M_{1}, 0\right)$, there exists $1 \leqslant j \leqslant r$ such that $\operatorname{Hom}_{A(z)}\left(X_{j}, M_{1}\right) \neq 0$. This shows that there is in $\bmod A(z)$ a path from $X_{j}$ to $Y$. 
On the other hand, there is in $\bmod A(z)$ a path from $Z$ to $N$, and $\Sigma_{A(z)}$ admits a path $\mathcal{D}=\mathcal{C}_{0} \rightarrow \cdots \rightarrow \mathcal{C}_{t}=\mathcal{C}$. Hence, the supports of $X_{j}$ and $N$ belong to the same connected component of $Q(z)$, because the radical $R(z)$ of $P(z)$ is separated. But then $\operatorname{Hom}_{A(z)}\left(R(z), X_{j}\right) \neq 0$ implies that $\operatorname{Hom}_{A(z)}\left(N, X_{j}\right) \neq 0$, and $\operatorname{so} \bmod A(z)$ admits an infinite path $Z \rightarrow Z_{1} \rightarrow \cdots \rightarrow N \rightarrow \cdots \rightarrow Y$. Thus, we get in $\Sigma_{A(z)}$ an oriented cycle $\mathcal{C} \rightarrow \cdots \rightarrow \mathcal{C}_{0} \rightarrow \cdots \rightarrow \mathcal{C}_{t}=\mathcal{C}$, a contradiction. Therefore, $\operatorname{Hom}_{A}(P(z), M)=0$, and $M$ is an $A(z)$-module.

For $x, y \in \mathcal{S}$, we set $x<^{*} y$ if and only if there is an infinite path

$$
M=Z_{0} \rightarrow Z_{1} \rightarrow \cdots \rightarrow Z_{s}=N,
$$

in $\bmod A(y)$ with $M$ an indecomposable direct summand of $R(x)$ and $N$ an indecomposable direct summand of $R(y)$. Then we have the following proposition.

(5.2) PROPOSITION. Assume that for each $x \in \mathcal{S}$ the component quiver $\Sigma_{A(z)}$ is directed. Then the transitive closure $\leqslant$ of $<^{*}$ is a partial order in $\mathcal{S}$.

Proof. Assume that $x_{0}<^{*} x_{1}<^{*} \ldots<^{*} x_{r}$ for some $x_{0}, x_{1}, \ldots, x_{r}$ in $\mathcal{S}$ and $r \geqslant 1$. We shall show that $x_{0} \neq x_{r}$. We may assume by induction that $x_{0}, x_{1}, \ldots x_{r-1}$ (respectively, $x_{1}, \ldots, x_{r}$ ) are pairwise different. By our assumption, for each $1 \leqslant i \leqslant r$, there exists an infinite path

$$
p_{i}: M_{i-1}=Z_{0}^{i} \rightarrow \cdots \rightarrow Z_{s_{i}}^{i}=N_{i}
$$

in $\bmod A\left(x_{i}\right)$ with $M_{i-1}$ an indecomposable direct summand of $R\left(x_{i-1}\right)$ and $N_{i}$ an indecomposable summand of $R\left(x_{i}\right)$. Denote by $\mathcal{D}_{0}, \ldots, \mathcal{D}_{r-1}$ the components of $\Gamma_{A\left(x_{r}\right)}$ containing the modules $M_{0}, \ldots, M_{r-1}$, respectively. Observe that, in order to prove our claim, it is enough to show that $\mathcal{D}_{0}$ is a full component of $\Gamma_{A}$. Indeed, then $\mathcal{D}_{0}$ consists of $A\left(x_{r}\right)$-modules and contains $P\left(x_{0}\right)$, because $\Gamma_{A}$ admits an arrow $M_{0} \rightarrow P\left(x_{0}\right)$. Hence $P\left(x_{0}\right) \neq P\left(x_{r}\right)$, and so $x_{0} \neq x_{r}$. Since $p_{r-1}$ is an infinite path from $M_{r-1}$ to $N_{r}$ in $\bmod A\left(x_{r}\right)$, applying Lemma 5.1 for $t=0, \mathcal{D}=\mathcal{D}_{r-1}, Z=M_{r-1}$ and $N=N_{r}$, we get that $\mathcal{D}_{r-1}$ is a full component of $\Gamma_{A}$. Hence, we may assume that $r \geq 2$. Now, since $x_{1}, \ldots, x_{r-1}$ are sources in $Q$ different from $x_{r}$, we conclude that $P\left(x_{1}\right), \ldots, P\left(x_{r-1}\right)$ are $A\left(x_{r}\right)$-modules. Then, for each $1 \leqslant i \leqslant r-1$, the component $\mathcal{D}_{i}$ admits arrows $M_{i} \rightarrow P\left(x_{i}\right)$ and $N_{i} \rightarrow P\left(x_{i}\right)$. Applying Lemma 5.1 again, we prove by descending induction on $i$ that, for each $r-1 \geqslant i \geqslant 1, p_{i-1}$ consists of $A\left(x_{r}\right)$-modules, $\mathcal{D}_{i-1}$ is a full component of $\Gamma_{A}$, and $\Sigma_{A\left(x_{r}\right)}$ admits a path $\mathcal{D}_{i-1} \rightarrow \cdots \rightarrow \mathcal{D}_{r-1}$. For $i=1$, we get that $\mathcal{D}_{0}$ is a full component of $\Gamma_{A}$. This finishes the proof.

\section{Proof of Theorem 4.1}

Let $A$ be a strongly simply connected algebra. We may assume that $A$ is connected. Clearly, (ii) implies (i). By Proposition 2.4, (i) implies (iii). Further each of the conditions (v) and (vi) implies that $\operatorname{rad}^{\infty}(M, M)=0$ for any $M \in \operatorname{ind} A$, and then 
(iv) holds. From Proposition 3.2 and Corollary 3.4, (iv) implies (iii). Moreover, the implication (vii) $\Rightarrow$ (ii) is a direct consequence of [3, Theorem 4.6], and Proposition 2.4(iii) (see also [50, Theorem 4.3]). Therefore, it remains to prove that (iii) implies (v), (vi) and (vii).

Assume that $A=K Q / I$ does not contain a convex subcategory which is $p g$-critical or hypercritical. Then, by Proposition 2.2, the Tits form $q_{A}$ of $A$ is weakly nonnegative. We shall prove (v), (vi) and (vii) by induction on the number of vertices in $Q$. We use the notations of Sections 4 and 5. Observe that, for any source $x$ of $Q$, the algebra $A(x)$ is strongly simply connected and does not contain a convex subcategory which is $p g$-critical or hypercritical. Hence, we may assume that, for any source $x$ in $Q, A(x)$ is a multicoil algebra, $\Sigma_{A(x)}$ is directed and every component of $\Gamma_{A(x)}$ is standard. Therefore, applying Proposition 5.2, we endow the set $\mathcal{S}$ of all sources in $Q$ by the partial order $\leqslant$ induced by $<^{*}$. Denote by $\mathcal{M}$ the set of all maximal elements in $\mathcal{S}$ with respect to $\leqslant$. We divide our proof into two main steps.

(1) Assume first that, for each $x \in \mathcal{M}, R(x)$ is a direct sum of indecomposable modules whose successors in $\Gamma_{A(x)}$ are all directing $A(x)$-modules. Fix $x \in \mathcal{M}$ and consider a decomposition $R(x)=M_{1} \oplus \cdots \oplus M_{r}$ of $R(x)$ into a direct sum of indecomposable $A(x)$-modules. For each $1 \leqslant i \leqslant r$, denote by $\mathcal{C}_{i}$ the component of $\Gamma_{A(x)}$ containing the module $M_{i}$, by $\mathcal{D}_{i}$ the full translation subquiver of $\mathcal{C}_{i}$ formed by all successors of $M_{i}$ in $\mathcal{C}_{i}$, and by $\mathcal{E}_{i}$ the full translation subquiver of $\mathcal{C}_{i}$ given by the remaining indecomposable modules. Clearly, $\mathcal{D}_{i}$ is closed under successors and $\mathcal{E}_{i}$ is closed under predecessors in $\mathcal{C}_{i}$. Moreover, by our assumption, each $\mathcal{D}_{i}$ consists entirely of directing $A(x)$-modules, and hence the number of $\tau_{A(x)}$-orbits in $\mathcal{D}_{i}$ is finite (see [53]). Further, let $\mathcal{C}$ be the component of $\Gamma_{A}$ containing $P(x), \mathcal{D}$ the full translation subquiver of $\mathcal{C}$ formed by all successors of modules $M_{1}, \ldots, M_{r}$ in $\mathcal{C}$, and $\mathcal{E}$ the full translation subquiver of $\mathcal{C}$ given by the remaining indecomposable $A$-modules. We shall show that $M_{1}, \ldots, M_{r}, P(x)$ are directing modules in $\bmod A$ and $\mathcal{D}$ contains all indecomposable projective $A$-modules being successors of modules $M_{1}, \ldots, M_{r}$ in $\bmod A$. Since the component quiver $\Sigma_{A(x)}$ is directed and $R(x)$ is separated, we have $\operatorname{Hom}_{A(x)}(R(x), Z)=0$ for any indecomposable $A(x)$ module $Z$ being predecessor in $\bmod A(x)$ of an indecomposable module lying in one of the subquivers $\mathcal{E}_{1}, \ldots, \mathcal{E}_{r}$. Applying now [39, (2.5)], to the one-point extension $A=A(x)[R(x)]$ we infer that $\mathcal{E}$ is the disjoint union of $\mathcal{E}_{1}, \ldots, \mathcal{E}_{r}$, and, if $\Gamma \rightarrow \cdots \rightarrow \mathcal{C}$ is a path in $\Sigma_{A}$ with $\Gamma \neq \mathcal{C}$, then $\Gamma$ is a full component from $\Gamma_{A(x)}$. Clearly, this implies that the modules $M_{1}, \ldots, M_{r}, P(x)$ are directing in $\bmod A$.

Let now $M_{i}=Y_{0} \rightarrow Y_{1} \rightarrow \cdots \rightarrow Y_{t}=P(b)$ be a path in $\bmod A$, for some $1 \leqslant i \leqslant r$. We claim that this path is finite, and hence $P(b)$ belongs to $\mathcal{D}$. Suppose that this is not the case. Then there is an infinite path in $\bmod A$ of the form $M_{i}=Y_{0} \rightarrow Y_{1} \rightarrow \cdots \rightarrow Y_{t} \rightarrow \cdots \rightarrow Y_{m}=P(c)$ with $c \in \mathcal{S}$. Clearly, $\operatorname{Hom}_{A}\left(P(c), M_{i}\right)=0$ because $c$ is source. Observe that then $m \geqslant 2$ and $\operatorname{Hom}_{A}\left(P(c), Y_{j}\right) \neq 0$ for some $1 \leqslant j \leqslant m-1$. Indeed, otherwise we have $x<{ }^{*} c$ which contradicts $x \in \mathcal{M}$. In particular, $P(c)$ is nondirecting in $\bmod A$, and by 
the above considerations $c \notin \mathcal{M}$. Hence $c<^{*} d$ for some $d \in \mathcal{S}$. Consequently, there is an infinite path $Z=Z_{0} \rightarrow Z_{1} \rightarrow \cdots \rightarrow Z_{s}=N$ in $\bmod A(d)$ with $Z$ an indecomposable direct summand of $R(c)$ and $N$ an indecomposable direct summand of $R(d)$. Since $Z$ and $P(c)$ belong to the same component, say $\mathcal{C}^{\prime}$, of $\Gamma_{A(d)}$ we infer by Lemma 5.1 that $Y_{0}, Y_{1}, \ldots, Y_{m}$ are $A(d)$-modules. In particular, $P(c)$ is nondirecting in $\bmod A(d)$. Since $A(d)$ is a multicoil algebra, we then conclude that $P(c)$ lies on a cycle in a coil $\Gamma^{\prime}$ of $\mathcal{C}^{\prime}$. Observe also that the path $Z=Z_{0} \rightarrow Z_{1} \rightarrow \cdots \rightarrow Z_{s}=N$ is not formed by directing modules lying on a sectional path of $\mathcal{C}^{\prime}$, because it is infinite (see [9]) and $\mathcal{C}^{\prime}$ is a standard multicoil. Then we infer that mod $A(d)$ admits an infinite path of the form

$$
M_{i}=Y_{0} \rightarrow Y_{1} \rightarrow \cdots \rightarrow Y_{m}=P(c) \rightarrow \cdots \rightarrow Z_{s}=N
$$

But this implies $x<^{*} d$, again a contradiction with $x \in \mathcal{M}$. Therefore, $\mathcal{D}$ contains any indecomposable projective $A$-module which is successor of a module $M_{i}, 1 \leqslant$ $i \leqslant r$, in $\bmod A$. The above $\operatorname{arguments~show~also~that,~if~} P(c)$ with $c \in \mathcal{S}$ is a successor of $P(x)$ in $\mathcal{D}$, then $c \in \mathcal{M}$. From the first part of our proof we know that, for any $c \in \mathcal{M}, P(c)$ is a directing $A$-module. Hence we may choose $x \in \mathcal{M}$ in such a way that any indecomposable proper successor of $P(x)$ in $\bmod A$ is not projective. For each $1 \leqslant i \leqslant r$, denote by $\Omega_{i}$ the set of all modules $X$ in $\mathcal{D}_{i}$ such that any path in $\mathcal{C}_{i}$ from $M_{i}$ to $X$ is sectional. Observe that each $\Omega_{i}$ is finite because the modules from $\Omega_{i}$ belong to pairwise different $\tau_{A(x)}$-orbits in $\mathcal{D}_{i}$. Further, put $\Omega=\left(\bigcup_{1 \leqslant i \leqslant r} \Omega_{i}\right) \cup\{P(x)\}$. Then, by the above discussion, we get that $\Omega$ is finite and consists of directing $A$-modules. Moreover, it follows from our new choice of $x$ that any module $U$ in $\mathcal{D}$ is of the form $\tau_{A}^{-l} V$ for some $V \in \Omega, l \geqslant 0$. Observe also that $\mathcal{D}$ has no oriented cycles. Indeed, since any proper successor of $P(x)$ in $\mathcal{D}$ is not projective, any cycle $U_{0} \rightarrow U_{1} \rightarrow \cdots \rightarrow U_{t} \rightarrow U_{0}$ in $\mathcal{D}$ gives a cycle $\tau_{A}^{l} U_{0} \rightarrow \tau_{A}^{l} U_{1} \rightarrow \cdots \rightarrow \tau_{A}^{l} U_{t} \rightarrow \tau_{A}^{l} U_{0}$, for some $l \geqslant 0$ passing through a module from $\Omega$, a contradiction because $\Omega$ consists of directing modules. Finally, suppose that there is in $\bmod A$ a cycle

$$
W=W_{0} \rightarrow W_{1} \rightarrow W_{2} \rightarrow \cdots \rightarrow W_{n} \rightarrow W_{n+1}=W,
$$

with $W$ from $\mathcal{D}$. Since $\mathcal{D}$ has no oriented cycle there exists $1 \leqslant q \leqslant n$ such that $W_{l} \in \mathcal{D}$ for any $l>q$ but $W_{q} \notin \mathcal{D}$. But then from the shape of $\mathcal{C}$ described above, we infer that $\bmod A$ admits a cycle of the form

$$
W=W_{0} \rightarrow W_{1} \rightarrow \cdots \rightarrow W_{q} \rightarrow L \rightarrow W_{q+1} \rightarrow \cdots \rightarrow W_{n+1}=W,
$$

with $L$ from $\Omega$. But it is not possible because $\Omega$ consists of directing modules. Therefore, $\mathcal{D}$ consists entirely of directing modules. We know that any module in $\mathcal{C}$ belongs either to $\mathcal{E}$ or to $\mathcal{D}$. Hence $\mathcal{C}$ either consists of directing modules or $\mathcal{C}$ is a multicoil (if one of $\mathcal{E}_{i}$ contains a coil). We shall show now that $\mathcal{C}$ is also standard. Observe that, since the modules $M_{i}, 1 \leqslant i \leqslant r$, are not predecessors of modules 
from $\mathcal{E}$ in $\bmod A$, we have $\operatorname{Hom}_{A}(X, Y)=0$ for all $X \in \mathcal{D}$ and $Y \in \mathcal{E}$. This implies that $\mathcal{C}$ is generalized standard. But $\mathcal{E}$ is standard, $\mathcal{D}$ is directed with finitely many $\tau_{A}$-orbits, so applying arguments as in [48], we conclude that $\mathcal{C}$ is in fact a standard component of $\Gamma_{A}$. We shall now describe $\Gamma_{A}$. Observe that any component $\mathcal{X}$ of $\Gamma_{A(x)}$ which has no module $M$ with $\operatorname{Hom}_{A(x)}(R(x), M) \neq 0$ remains a full component in $\Gamma_{A}$, by [39, (2.5)]. Hence, we must describe the components of $\Gamma_{A}$ containing successors of $P(x)$ in $\bmod A$. Consider the right stable part $\mathcal{D}_{r}$ of $\mathcal{D}$ obtained from $\mathcal{D}$ by removing the $\tau_{A}$-orbits of injective modules. If $\mathcal{D}_{r}$ is empty, $\mathcal{C}$ is the unique component of $\Gamma_{A}$ which is not a component of $\Gamma_{A(x)}$. Clearly, then $A=A(x)[B(x)]$ is a multicoil algebra, $\Sigma_{A}$ is directed and every component of $\Gamma_{A}$ is standard. Assume that $\mathcal{D}_{r}$ is not empty. The $\mathcal{D}$ admits a full translation subquiver $\mathcal{F}$ of $\mathcal{D}_{r}$ which is closed under successors in $\mathcal{D}$ (hence in $\mathcal{C}$ ), contains all but finitely many modules of $\mathcal{D}$, and is a disjoint union of translation quivers of the form $\mathcal{F}_{j}=(-\mathbb{N}) \Delta_{j}, 1 \leqslant j \leqslant t$, for some finite quivers $\Delta_{1}, \ldots, \Delta_{t}$ without oriented cycles (see [27] for the shape of right stable quivers without oriented cycles). Fix $1 \leqslant j \leqslant t$. Since $\mathcal{F}_{j}$ consists of directing modules, applying the dual of Proposition 2.4 in [53], we conclude that there exists a hereditary algebra $H_{j}$ of type $\Delta_{j}$ and a tilting $H_{j}$-module $T_{j}$ without preprojective direct summands such that the tilted algebra $B_{j}=\operatorname{End}_{H_{j}}\left(T_{j}\right)$ is a convex subcategory of $A$ and $\mathcal{F}_{j}$ is a full translation subquiver of a connecting component $\mathcal{P}_{j}$ of $\Gamma_{B_{j}}$ which is closed under successors. Since $q_{A}$ is weakly nonnegative, we conclude that $q_{B_{j}}$ is weakly nonnegative, and hence, by Proposition $2.8, \Delta_{j}$ is an Euclidean quiver. In particular, $\mathcal{P}_{j}$ is the preprojective component of $\Gamma_{B_{j}}$. Recall that (see Section 1) $\Gamma_{B_{j}}$ consists of $\mathcal{P}_{j}$, a $\mathbb{P}_{1}(K)$-family $\mathcal{T}_{j}$ of tubes without projective modules and a preinjective component $\mathcal{I}_{j}$. Moreover, all components of $\Gamma_{B_{j}}$ are standard, $\Sigma_{B_{j}}$ is directed, and $B_{j}$ is a multicoil algebra. Now, since $P(x)$ has no proper projective successors in $\bmod A$, it follows that any component $\mathcal{X}$ in $\Gamma_{A}$ different from $\mathcal{C}$ and containing a successor of $P(x)$ in $\bmod A$ is a component from the family $\bigvee_{1 \leqslant j \leqslant t}\left(\mathcal{T}_{j} \vee \mathcal{I}_{j}\right)$. We know also that $\operatorname{Hom}\left(\mathcal{T}_{p} \vee \mathcal{I}_{p}, \mathcal{T}_{q} \vee \mathcal{I}_{q}\right)=0$ for $p \neq q$. Therefore, we get that $\Sigma_{A}$ is directed, all components of $\Gamma_{A}$ are standard, and $A$ is a multicoil algebra.

(2) Assume now that, for some $y \in \mathcal{M}$ and an indecomposable direct summand $U$ of $R(y)$, there exists a path $U=U_{0} \rightarrow U_{1} \cdots \rightarrow U_{r}=Z$ in $\Gamma_{A(y)}$ with $Z$ nondirecting. We shall show that then there exists $x \in \mathcal{M}$ such that $R(x)$ admits a nondirecting indecomposable direct summand $M$. If $U$ is nondirecting, we set $x=y$ and $M=U$. Assume $U$ is directing. Since $A(y)$ is a multicoil algebra, $Z$ lies in a coil $\Omega$ of a standard multicoil $\mathcal{X}$ of $\Gamma_{A(y)}$. By [4, Lemma 3.3], we may assume that the coil $\Omega$ is proper, that is, any module from $\Omega$ lies on a cycle in $\Omega$. Observe that $\Omega$ contains at least one projective module, because there is in $\mathcal{X}$ a path from the directing module $U$ to the module $Z$ lying in $\Omega$. Further, it follows from the structure of standard coils that, if $\operatorname{Hom}_{A(y)}(P(a), P(b)) \neq 0$ for $P(a)$ from $\Omega$ and $P(b)$ from $\mathcal{X}$, then $P(b)$ lies in $\Omega$. Hence, we infer that there exists in $\mathcal{X}$ a path $U=U_{0} \rightarrow U_{1} \rightarrow \cdots \rightarrow U_{s} \rightarrow P(x)$ such that $P(x)$ lies in $\Omega$ and $\operatorname{Hom}_{A(y)}(P(x), P(c))=0$ for any projective module $P(c)$ from $\mathcal{X}$ nonisomorphic 
to $P(x)$. We claim that $x \in \mathcal{M}$. First we show that $x \in \mathcal{S}$. Suppose $x \notin \mathcal{S}$. Then we have in $\bmod A(y)$ a path $P(x) \rightarrow P\left(e_{1}\right) \rightarrow \cdots \rightarrow P\left(e_{r}\right)=P(z)$ with $z \in \mathcal{S}$. By our choice of $x$ we infer that $P\left(e_{1}\right)$ does not belong to $\mathcal{X}$. Then, since $\Sigma_{A(y)}$ is directed, we have $\operatorname{Hom}_{A(y)}\left(P(z), U_{l}\right)=0$ for any $0 \leqslant l \leqslant s$. Therefore we get an infinite path in $\bmod A(z)$ of the form

$$
U=U_{0} \rightarrow U_{1} \rightarrow \cdots \rightarrow U_{s} \rightarrow P(x) \rightarrow \cdots \rightarrow P\left(e_{r-1}\right) \rightarrow V
$$

where $V$ is an indecomposable direct summand of $R(z)$. But then $y<^{*} z$, a contradiction because $y \in \mathcal{M}$. Hence, $x \in \mathcal{S}$. Suppose now that $x<^{*} w$ for some $w \in \mathcal{S}$. Then $\bmod A(w)$ admits an infinite path

$$
N=Z_{0} \rightarrow Z_{1} \rightarrow \cdots \rightarrow Z_{q}=T
$$

where $N$ is an indecomposable direct summand of $R(x)$ and $T$ an indecomposable direct summand of $R(w)$. Denote by $\mathcal{Y}$ and $\mathcal{Z}$ the components of $\Gamma_{A(w)}$ containing the modules $N$ and $T$, respectively. Clearly, $\mathcal{Y} \neq \mathcal{Z}$, because $\Sigma_{A(w)}$ is directed. Applying now Lemma 5.1 we conclude that $\mathcal{Y}$ is in fact a component of $\Gamma_{A}$ and for any path in $\bmod A$ with target lying in $\mathcal{Y}$ its source is an $A(w)$-module. Let $p$ be minimal such that $Z_{p}$ does not belong to $\mathcal{Y}$. Then $\operatorname{rad}^{\infty}\left(Z_{p-1}, Z_{p}\right) \neq 0$, and we have in $\bmod A(w)$ paths

$$
N=Z_{0} \rightarrow \ldots \rightarrow Z_{p-1} \rightarrow W_{1} \rightarrow \cdots \rightarrow W_{m} \rightarrow Z_{p} \rightarrow \cdots \rightarrow Z_{q}=T,
$$

for all $m \geqslant 1$. On the other hand, since $N$ is a direct predecessor of $P(x)$ and $P(x)$ lies in a proper coil $\Delta$ of the standard multicoil $\mathcal{Y}$ of $\Gamma_{A(w)}$, we infer that $P(x)$ is a predecessor of $W_{m}$ in $\Delta$ for a sufficiently large $m$. We then conclude that $\bmod A(w)$ admits an infinite path $U=U_{0} \rightarrow \cdots \rightarrow P(x) \rightarrow \cdots \rightarrow W_{m} \rightarrow Z_{p} \rightarrow$ $\cdots \rightarrow Z_{q}=T$, and so $y<{ }^{*} w$. This contradiction proves that $x \in \mathcal{M}$. Let $M$ be a nondirecting indecomposable direct summand of $R(x)$ and $B$ the connected part of $A(x)$ such that $M$ is a $B$-module. Since $B$ is a convex subcategory of $A(x)$, the conditions (v), (vii) and (viii) hold for $B$. Further, $M$ is nondirecting in $\bmod B$, and so it belongs to a proper coil $\Gamma$ of a standard multicoil $\mathcal{C}$ of $\Gamma_{B}$. We shall show that the vector space category $\operatorname{Hom}_{B}(M, \mathcal{C})$, given by the objects $\operatorname{Hom}_{B}(M, X)$ with $X$ from $\mathcal{C}$, is the $K$-linear category of one of the following partially ordered sets:

(a) $\operatorname{Hom}_{B}\left(M, X_{0}\right) \rightarrow \operatorname{Hom}_{B}\left(M, X_{1}\right) \rightarrow \operatorname{Hom}_{B}\left(M, X_{2}\right) \rightarrow \cdots$

$$
\begin{aligned}
\operatorname{Hom}_{B}\left(M, Y_{t}\right) \leftarrow \cdots \leftarrow & \operatorname{Hom}_{B}\left(M, Y_{1}\right) \\
& \operatorname{Hom}_{N}\left(M, X_{0}\right) \rightarrow \operatorname{Hom}_{B}\left(M, X_{1}\right) \rightarrow \cdots
\end{aligned}
$$

with $t \geqslant 1$ and $X_{0}$ injective, 
(c)

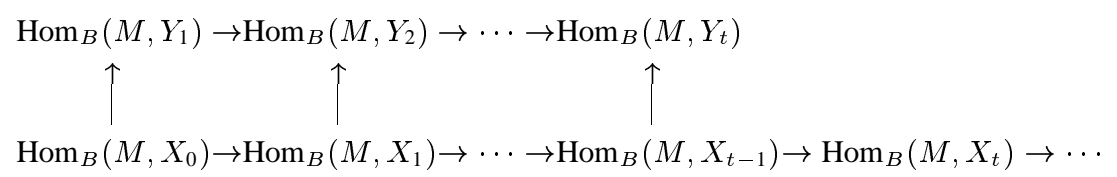

with $t \geqslant 2$ and $X_{t-1}$ injective,

where $M=X_{0}, X_{1}, X_{2}, \ldots$ lie on a unique infinite sectional path $X_{0} \rightarrow X_{1} \rightarrow$ $X_{2} \rightarrow \cdots$ in $\mathcal{C}$ with source $M=X_{0}$, and $Y_{1}, \ldots Y_{t}$ are directing modules lying on a sectional path $Y_{1} \rightarrow Y_{2} \cdots \rightarrow Y_{t}$ in $\mathcal{C}$. Moreover, we claim that, if $R(x)$ is decomposable, then $A(x)=B \times D, R(x)=M \oplus N$, where $D$ is connected, $N$ is an indecomposable $D$-module, $\operatorname{Hom}_{B}(M, \mathcal{C})$ is of the form (a), and the vector space category $\operatorname{Hom}_{D}(N$, ind $D)$ is the $K$-linear category of a partially ordered set

$$
\operatorname{Hom}_{D}\left(N, Y_{1}\right) \rightarrow \operatorname{Hom}_{D}\left(N, Y_{2}\right) \rightarrow \cdots \rightarrow \operatorname{Hom}_{D}\left(N, Y_{t}\right)
$$

with $t \geqslant 1$ and $N=Y_{1}, \ldots, Y_{t}$ directing modules lying on a unique sectional path $Y_{1} \rightarrow \cdots \rightarrow Y_{t}$ in $\Gamma_{D}$.

We divide our considerations into several steps.

$(\alpha)$ Suppose that $\mathcal{C}=\Gamma$ is a stable tube. Then $C=\operatorname{supp} \Gamma$ is a convex subcategory of $B$ (see [2, (3.1)]) whose Auslander-Reiten quiver admits a sincere stable tube. Since, by our assumption, $C$ is also a multicoil algebra (hence cycle-finite), we get from [3, (4.1)] (see also [50, (4.1)]), that $C$ is either critical or tubular. Clearly, $E=C[M]$ is also a convex subcategory of $A$, and hence $q_{E}$ is weakly nonnegative and $E$ does not contain a $p g$-critical convex subcategory. Then, by Proposition 2.5, $C$ is critical. Applying now Proposition 2.6, we infer that $M$ is a simple regular module, and so $E$ is a tubular extension of $C$. Therefore, $E$ is either tubular or representation-infinite tilted algebra of Eulidean type with a complete slice in the preinjective component, because $q_{E}$ is weakly nonnegative (Proposition 2.3). Clearly, in this case, $\mathcal{C}$ admits a unique sectional path $M=X_{0} \rightarrow X_{1} \rightarrow X_{2} \rightarrow \cdots$ consisting of arrows pointing to infinity, and $\operatorname{Hom}_{B}(M, \mathcal{C})$ is of type (a).

( $\beta$ ) Assume that $\mathcal{C}$ is not a stable tube. Then by [4, (5.9)], there exists a critical convex subcategory $C$ of $B$ and a stable tube $\mathcal{T}$ in $\Gamma_{C}$ such that $\Lambda=\operatorname{supp} \Gamma$ is obtained from $C$ by a sequence of admissable operations of types (ad 1), (ad 2), $(\operatorname{ad} 3),\left(\operatorname{ad~} 1^{*}\right),\left(\operatorname{ad} 2^{*}\right)$ or $\left(\operatorname{ad~} 3^{*}\right), \Gamma_{\Lambda}$ admits a coil $\Gamma^{\prime}$ obtained from the stable tube $\mathcal{T}$ be the corresponding sequence of admissible operations, and such that $\Gamma$ is the full translation subquiver $\Gamma_{\gamma}^{\prime}$ of $\Gamma^{\prime}$ consisting of all modules lying on cycles in $\Gamma^{\prime}$ (see [4, Section 3] for details). Then $\Lambda=\operatorname{supp} \Gamma$ is a coil enlargement of $C$ in the sense of [5]. Moreover, $\Lambda$ is a convex subcategory of $B$. Applying now [5, (3.5)], we infer that there is a unique tubular extension $E=\Lambda^{+}$of $C$ which is a convex subcategory of $\Lambda$ and such that $\Lambda$ can be obtained from $E$ by a sequence of one-point coextensions of types $\left(\operatorname{ad~} 1^{*}\right),\left(\operatorname{ad} 2^{*}\right)$, or $\left(\operatorname{ad~} 3^{*}\right)$. Furthermore, there is a ray tube $\mathcal{T}^{+}$in $\Gamma_{E}$ such that $\Gamma^{\prime}$ can be obtained from $\mathcal{T}^{+}$by the corresponding 
sequence of admissible operations of types $\left(\operatorname{ad} 1^{*}\right),\left(\operatorname{ad} 2^{*}\right)$, or $\left(\operatorname{ad~} 3^{*}\right)$, being coray insertions. Since $M$ lies in $\Gamma=\Gamma_{\gamma}^{\prime}$, there exists an infinite sectional path

$$
\Sigma: M=X_{0} \rightarrow X_{1} \rightarrow X_{2} \rightarrow X_{3} \rightarrow \cdots
$$

in $\Gamma$ consisting of arrows pointing to infinity. Further, since $\Gamma^{\prime}$ is obtained from the ray tube $\mathcal{T}^{+}$by a sequence of coray insertions (operations of types $\left(\operatorname{ad} 1^{*}\right),\left(\operatorname{ad} 2^{*}\right)$, $\left(\right.$ ad $\left.\left.3^{*}\right)\right)$, we get that, for infinitely many $i \geqslant 0, X_{i}$ is an $E$-module. We claim now that $\operatorname{Hom}_{B}(M, \mathcal{C})$ does not contain a full subcategory which is the $K$-linear category of the partially ordered set

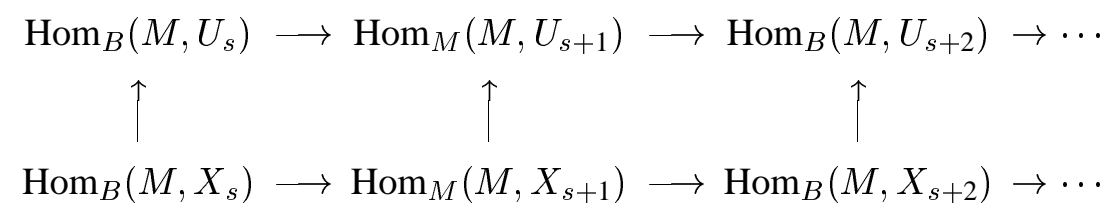

$s \geqslant r$, with $U_{s}, U_{s+1}, \ldots$, lying on an infinite sectional path $U_{s} \rightarrow U_{s+1} \rightarrow$ $U_{s+2} \rightarrow \cdots$ in $\Gamma^{\prime}$ which is parallel to $X_{s} \rightarrow X_{s+1} \rightarrow X_{s+2} \rightarrow \cdots$. Suppose that this is not the case. Then by the above remark $\mathcal{T}^{+}$admits a subquiver of the form

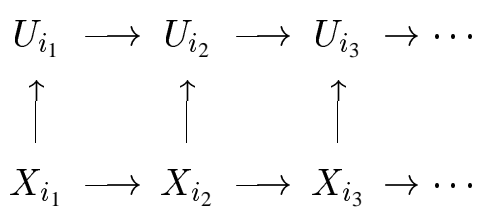

where $M^{\prime}=X_{i_{1}}$ is the restriction of $M=X_{0}$ to $E=\Lambda^{+}$. Moreover, $E^{\prime}=E\left[M^{\prime}\right]$ is a convex subcategory of $A$, and hence is strongly simply connected. Applying now Proposition 2.6 to the one-point extension $E^{\prime}=E\left[M^{\prime}\right]$ we get that either $E^{\prime}$ contains a convex $p g$-critical subcategory or $q_{E^{\prime}}$ is not weakly nonnegative, which contradicts our assumptions on $A$. The proved fact shows that $\Sigma$ is a unique infinite sectional path in $\mathcal{C}$ with source $M$. We claim now that if $X_{i} \rightarrow P$ is an arrow in $\mathcal{C}$ with $P$ projective then $P$ lies on $\Sigma$. Suppose that $X_{i} \rightarrow P$, for some $i \geqslant 0$, is an arrow in $\mathcal{C}$ with $P$ projective not lying on $\Sigma$. From the above remarks we know that then $\mathcal{C}$ has no infinite sectional path with source $P$ and parallel to $\Sigma$. Hence, there is $j>i$ such that $X_{i}, \ldots, X_{j-1}$ are noninjective but $X_{j}$ is injective. Applying now Lemma $(3+3+2)$ in $[2,(2.1)]$, we obtain an exact sequence

$$
0 \rightarrow X_{i} \rightarrow P \oplus X_{j} \oplus V \rightarrow \tau_{B}^{-} X_{j-1} \rightarrow 0 .
$$

On the other hand we have $\operatorname{dim}_{K} X_{i}<\operatorname{dim}_{K} P$ and $\operatorname{dim}_{K} \tau_{B}^{-} X_{j-1}<\operatorname{dim}_{K} X_{j}$. This contradiction proves our claim. We recall also that by [5, (3.5)], there is a unique tubular coextension $F=\Lambda^{-}$of $C$ and a coray tube $\mathcal{T}^{-}$in $\Gamma_{F}$ such that $\Lambda$ is obtained from $F$ by a sequence of admissible operations of types $(\operatorname{ad} 1),(\operatorname{ad} 2)$ or (ad 3), and $\Gamma^{\prime}$ is obtained from $\mathcal{T}^{-}$by the corresponding sequence of admissible 
operations (ray insertions). Hence, if $X_{i} \rightarrow Z$ is an arrow in $\mathcal{C}$ with $Z \not X_{i+1}$ then $Z$ is from $\mathcal{T}^{-}$. In particular, any module $X_{i}, i \geqslant 0$, has at most two direct successors in $\mathcal{C}$. Therefore, if $M$ has exactly one direct successor, then the vector space category $\operatorname{Hom}_{B}(M, \mathcal{C})$ is of type (a).

$(\gamma)$ Assume now that $\mathcal{C}$ admits a full translation subquiver

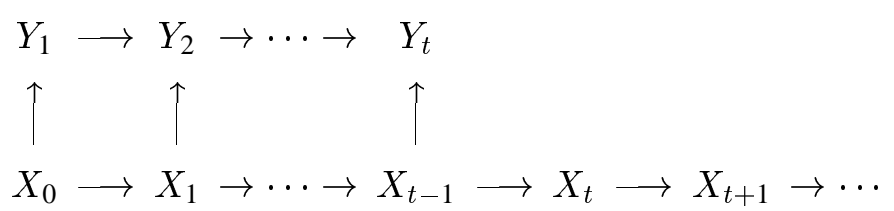

with $t \geqslant 2$ and $X_{t-1}$ injective. We claim that then $R(x)=M, \operatorname{Hom}_{B}(M, \mathcal{C})$ is of the form (c), induced by the above subquiver of $\mathcal{C}$, and no module $Y_{j}, 1 \leqslant j \leqslant t$, is predecessor of a projective module in $\mathcal{C}$. Suppose that $R(x) \not M$ or $\mathcal{C}$ admits an arrow $Y_{j} \rightarrow W$ with $1 \leqslant j \leqslant t$ and $W \not Y_{j+1}$ (possibly $W$ is projective). We know that the modules $Y_{1}, \ldots, Y_{t}$ are from the coray tube $\mathcal{T}^{-}$, and so their supports are in one coextension branch of the tubular coextension $F$ of $C$. We look now on the supports of the modules $Y_{1}, \ldots, Y_{t}$ and apply the rules for the Auslander-Reiten sequences in the module categories over special biserial simply connected algebras described in [54, p. 180] (see also [37]). Then a simple analysis (see the proof of Lemma 3.5 in [3]) shows that $A=A(x)[R(x)]$ admits a convex subcategory of a form described in Corollary 2.7. This contradicts our assumption on $A$. Hence $R(x)=M$ and $\operatorname{Hom}_{B}(M, \mathcal{C})$ is of the required form (c). Suppose now that one of the modules $Y_{1}, \ldots, Y_{t}$ is a predecessor of a projective module in $\mathcal{C}$. Then $\mathcal{C}$ admits a path of the form

$$
Y_{j}=V_{0} \rightarrow V_{1} \rightarrow \cdots \rightarrow V_{p-1} \rightarrow V_{p}=P(y),
$$

with $j \geqslant 2, p \geqslant 2, V_{1}=\tau_{B}^{-} Y_{j-1}$ and $V_{0}, V_{1}, \ldots, V_{p}$ directing in $\bmod B$. Let

$$
P(y)=P\left(y_{0}\right) \rightarrow P\left(y_{1}\right) \rightarrow \cdots \rightarrow P\left(y_{l}\right)=P(z),
$$

be a path in $\bmod B$ with $z \in \mathcal{S}$. We then have a path

$$
M=X_{0} \rightarrow \cdots \rightarrow X_{j-1} \rightarrow V_{0} \stackrel{\varphi}{\rightarrow} V_{1} \rightarrow \cdots \rightarrow V_{p-1} \rightarrow \cdots \rightarrow L
$$

with $L$ and indecomposable direct summand of $R(z)$. Since $V_{0}, \ldots, V_{p-1}$ are directing modules in $\bmod B$, we infer that in fact it is a path in $\bmod A(z)$. We claim that $\varphi$ belongs to $\operatorname{rad}^{\infty}(\bmod A(z))$. Indeed, this follows from Lemma 3.1 if we take $R=X_{0}, Y=Y_{j}, Z=\tau^{-} Y_{j-1}, W_{i}=X_{t+i}, i \geqslant 1, f_{i}: W_{i} \rightarrow W_{i+1}, i \geqslant 1$, arbitrary irreducible maps and $g: R \rightarrow Y, f_{0}: R \rightarrow W_{1}$ the compositions of the corresponding irreducible maps. Hence we get an infinite path in $\bmod A(z)$ from $M$ to $L$, and so $x<z$. But this is a contradiction with the maximality of $x$ in $\mathcal{S}$. Therefore, the modules $Y_{1}, \ldots, Y_{t}$ have no projective successors in $\mathcal{C}$. 
$(\delta)$ Assume now that $\operatorname{Hom}_{B}(M, \mathcal{C})$ is of the form (a) but $R(x)=M \oplus N$ with $N \neq 0$. We know that the restriction $M^{\prime}$ of $M$ to the tubular extension $E=\Lambda^{+}$ of $C$ is nonzero. Then applying Corollary 2.7 we infer that $N$ is indecomposable, and moreover uniserial. Hence, $A(x)=B \times D$ where $D$ is connected and $N$ is in $\bmod D$. Let $\mathcal{D}$ be the component of $\Gamma_{D}$ containing $N$. We claim that there is a sectional path

$$
N=Y_{1} \rightarrow Y_{2} \rightarrow \cdots \rightarrow Y_{t}
$$

in $\mathcal{D}$ formed by directing modules such that the vector space category $\operatorname{Hom}_{D}(N$, ind $D$ ) is the $K$-linear category of the partially ordered set

$$
\operatorname{Hom}_{D}\left(N, Y_{1}\right) \rightarrow \operatorname{Hom}_{D}\left(N, Y_{2}\right) \rightarrow \cdots \rightarrow \operatorname{Hom}_{D}\left(N, Y_{t}\right)
$$

Since $N$ is uniserial and $D$ is strongly simply connected, as a convex subcategory of $A$, the support of $N$ is given by a convex line in $Q_{D}$ of the form $a_{1} \rightarrow a_{2} \rightarrow$ $\cdots \rightarrow a_{r}$. Let $G$ be the maximal convex subcategory of $D$ which is the bound quiver algebra of a finite connected bound subquiver of the following infinite tree

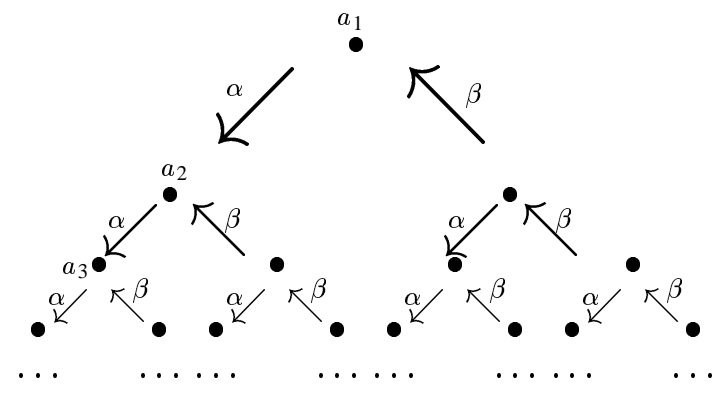

bound by all possible relations $\beta \alpha=0$, and $N$ is the projective $G$-module $P_{G}\left(a_{1}\right)$. It is known (see $[49,(4.4)]$ ) that $G$ is a tilted algebra of Dynkin $A_{t}$ and $\Gamma_{G}$ admits a complete section (slice) $\Theta$ of the form

$$
N=Y_{1} \rightarrow Y_{2} \rightarrow \cdots \rightarrow Y_{t}
$$

Using the maximality of $G$, our assumptions on $A$, and applying Corollary 2.7 again, we infer that, if $G[V]$ is a one-point extension of $G$ inside $D$ with $V$ indecomposable, then $V$ is a proper successor of a module $Y_{i}$ and does not lie on $\Theta$. In particular, $\operatorname{Hom}_{D}(N$, ind $D)$ is the required $K$-linear category of a finite linear partially ordered set. The maximality of $x$ in $\mathcal{S}$ implies also that any successor of $N$ in $\Gamma_{D}$ is a $G$-module. Indeed, if this is not the case, then there is in $\bmod D$ a path of the form

$$
Y_{j}=V_{0} \stackrel{\varphi}{\rightarrow} V_{1} \rightarrow \cdots \rightarrow V_{p-1} \rightarrow P\left(y_{1}\right) \rightarrow \cdots \rightarrow P\left(y_{l}\right) \rightarrow L
$$


where $j \geqslant 2, V_{1}=\tau_{G}^{-} Y_{j-1}, V_{0}, \ldots, V_{p-1}$ are directing $D$-modules (in fact $G$ modules) and $L$ is an indecomposable direct summand of $R(z)$ for some $z \in \mathcal{S}$. Clearly, then it is a path in $\bmod A(z)$. We claim that $\varphi \in \operatorname{rad}^{\infty}(\bmod A(z))$. This follows from Lemma 3.1 if we put $R=R(x), Y=V_{0}, Z=V_{1}, W_{i}=X_{i}$ for $i \geqslant 1$, and take for $f_{i}: W_{i} \rightarrow W_{i+1}$ arbitrary irreducible maps, for $f_{0}: R \rightarrow$ $W_{1}=X_{1}$ the composition of the projection $R=M \oplus N \rightarrow X_{0}=M$ with an irreducible map $X_{0} \rightarrow X_{1}=W_{1}$, and for $g: R \rightarrow Y$ the composition of the projection $R=M \oplus N \rightarrow N$ with irreducible maps $Y_{1} \rightarrow \cdots \rightarrow Y_{j}$. Since $\operatorname{Hom}_{A(x)}\left(P(z), Y_{j}\right)=0$ and $\operatorname{Hom}_{A(x)}\left(P(z), X_{i}\right)=0$ for all $i \geqslant 1$ we get from the proof of Lemma 3.1 that in fact $\varphi \in \operatorname{rad}^{\infty}(\bmod A(z))$. Therefore, we have in $\bmod A(z)$ an infinite path from $N$ to $L$, a contradiction with the maximality of $x$ in $\mathcal{S}$.

$(\sigma)$ Assume now that $M$ is injective and has two direct successors in $\mathcal{C}$. Applying Corollary 2.7 we infer that then $R(x)=M$. Let $Y_{1}$ be the direct successor of $M$ in $\mathcal{C}$ different from $X_{1}$. Then $Y_{1}$ is a module from $\mathcal{T}^{-}$. Observe that $M$ is not necessarily an $F$-module but its restriction to $F$ is an injective $F$-module having two direct successors in $\mathcal{T}^{-}$. Let

$$
Y_{1}=U_{1} \rightarrow U_{2} \rightarrow \cdots \rightarrow U_{r}
$$

be the maximal sectional path in $\mathcal{T}^{-}$with source $Y_{1}$. Clearly, it consists of injective $F$-modules. Applying Corollary 2.7 again we infer that none of modules $U_{1}, \ldots, U_{r-1}$ is a direct summand of the radical of a projective module inside $B$. Suppose now that there is in $\mathcal{T}^{-}$a sectional path

$$
V=V_{0} \rightarrow V_{1} \rightarrow \cdots \rightarrow V_{s}=U_{k}
$$

with $1 \leqslant k \leqslant r-1$ such that $V$ is a summand of the radical of a projective module $P(d)$ in $B$. We may assume that $k$ is minimal with this property. Since $F=\Lambda^{-}$is a tubular coextension of $C$, the supports of all successors of $V$ in $\mathcal{T}^{-}$ are contained in one (coextension) branch of $F$. Looking on the support of $V$, and applying Corollary 2.7 and the formulas for the Auslander-Reiten sequences in module categories over special biserial simply connected algebras (see [54], [37]), we conclude that the vector space category $\operatorname{Hom}_{F}(V$, ind $F)$ is the $K$-linear category of one of the following finite partially ordered sets:

$$
\begin{aligned}
& \operatorname{Hom}_{F}\left(V, V_{0}\right) \rightarrow \operatorname{Hom}_{F}\left(V, V_{1}\right) \rightarrow \cdots \rightarrow \operatorname{Hom}_{F}\left(V, V_{s}\right) \\
& \operatorname{Hom}_{F}\left(V, W_{l}\right) \leftarrow \cdots \rightarrow \operatorname{Hom}_{F}\left(V, W_{1}\right) \leftarrow \operatorname{Hom}_{F}\left(V, V_{0}\right) \rightarrow \cdots \rightarrow
\end{aligned}
$$$$
\operatorname{Hom}_{F}\left(V, V_{s}\right)
$$

with $l \geqslant 1$ and $V=V_{0}$ injective, $\left(\mathrm{c}^{\prime}\right)$ 


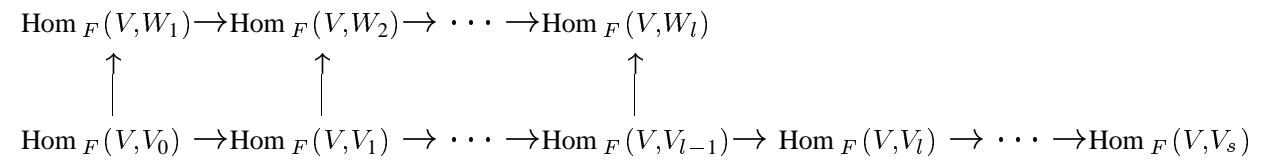

with $2 \leqslant l \leqslant s$ and $V_{l-1}$ injective.

Moreover, we conclude as above that, if the radical $R(d)$ of $P_{A}(d)$ is decomposable, then $\operatorname{Hom}_{F}(V$,ind $F)$ is of the form $\left(\mathrm{a}^{\prime}\right), R(d)=V \oplus W$, where $W$ is a uniserial module whose support is the $K$-linear category $H$ a linear quiver $l \rightarrow l-1 \rightarrow \cdots \rightarrow$ $2 \rightarrow 1, l \geqslant 1$. Then, applying [4, (2.1)], and [37], we infer that, for $F^{\prime}=F[V]$ or $F^{\prime}=(F \times H)[V \oplus W]$, the component of $\Gamma_{F^{\prime}}$ containing $P(d)$ is obtained from the coray tube $\mathcal{T}^{-}$by inserting a rectangle and glueing with $\mathcal{T}^{-}$as follows:

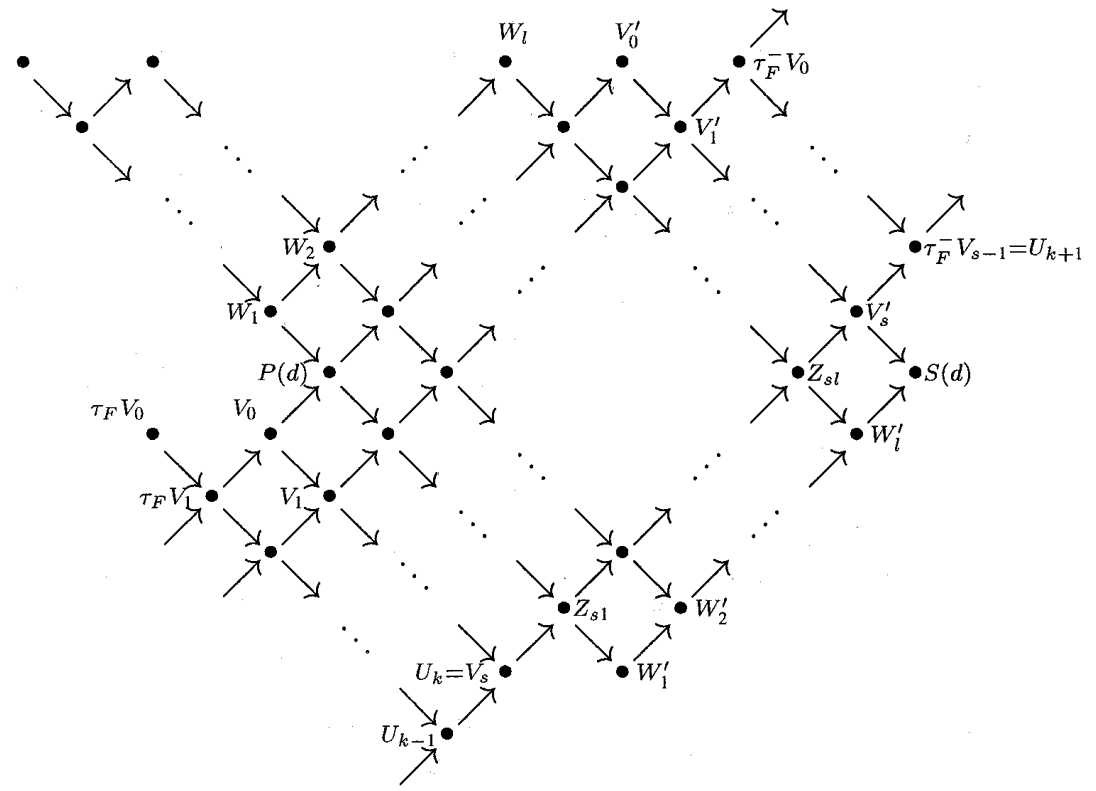

if $\operatorname{Hom}_{F}(V$,ind $F)$ is of type $\left(\mathrm{a}^{\prime}\right)$ and $R(d)$ decomposable;

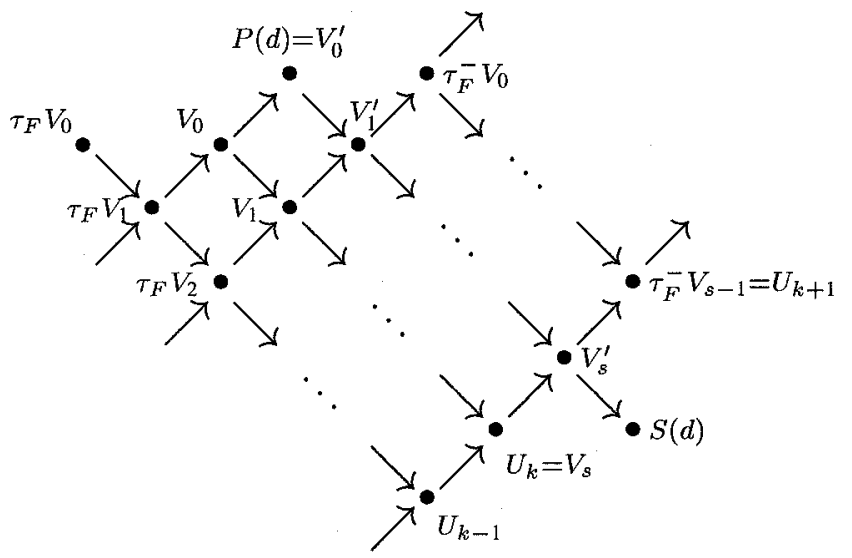


if $\operatorname{Hom}_{F}(V$, ind $F)$ is of type $\left(\mathrm{a}^{\prime}\right)$ and $R(d)$ indecomposable;

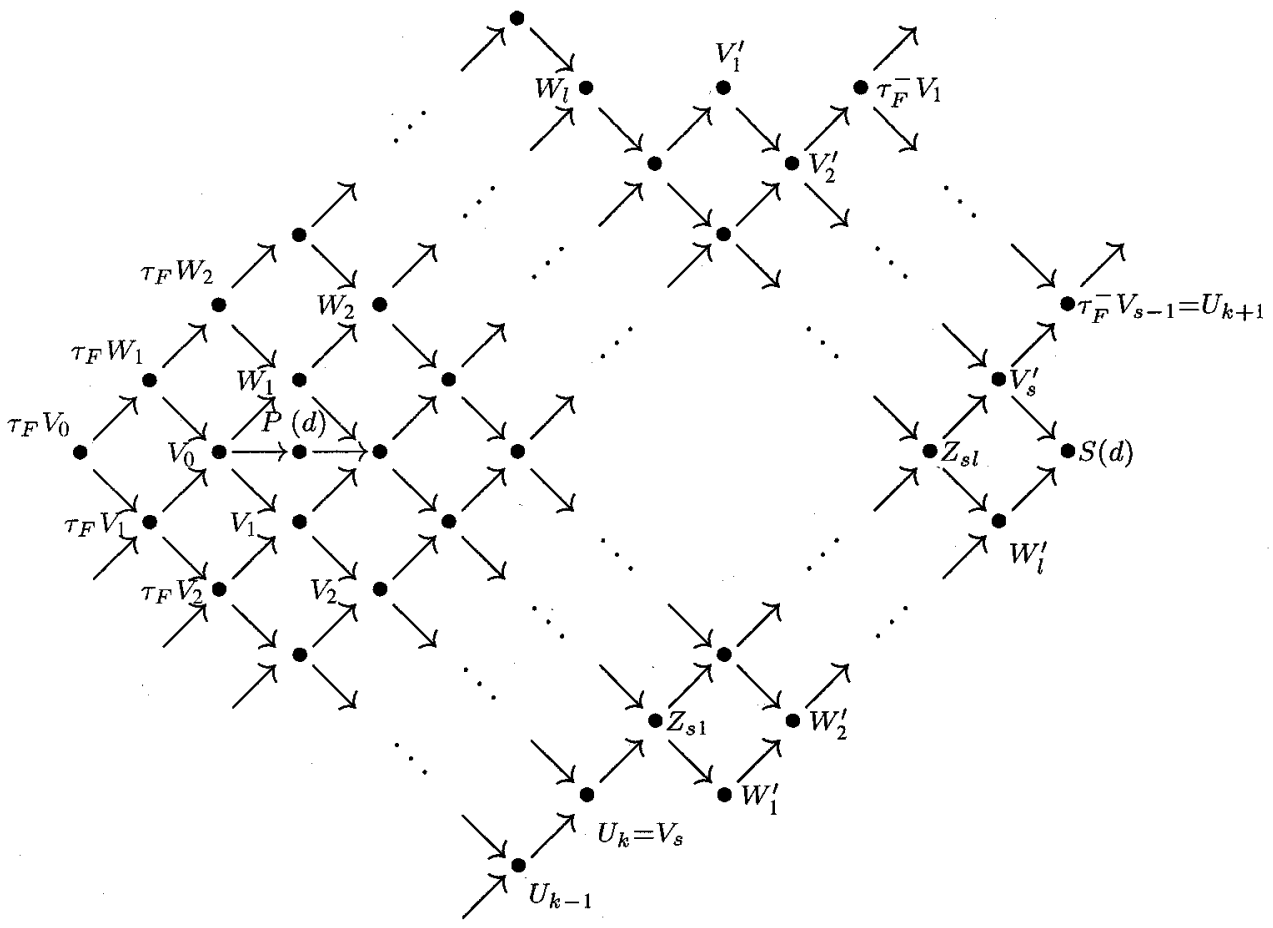

if $\operatorname{Hom}_{F}(V$,ind $F)$ is of type $\left(\mathrm{b}^{\prime}\right)$;

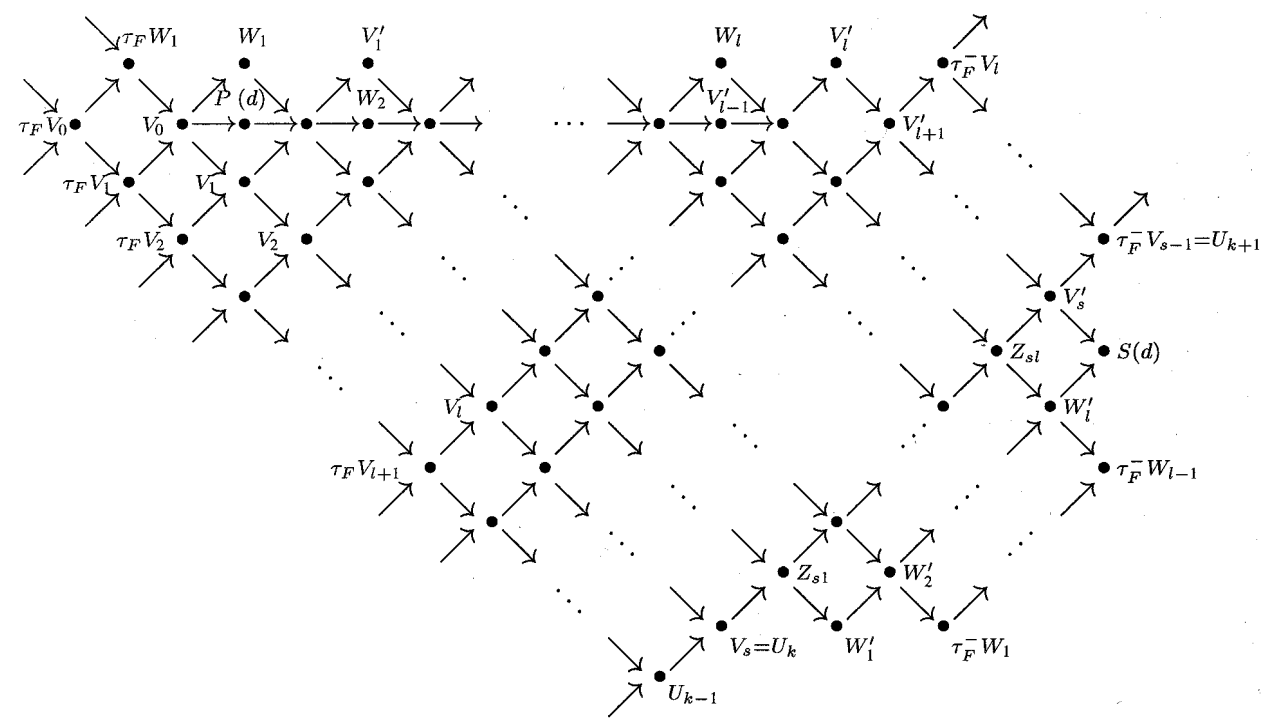


if $\operatorname{Hom}_{F}(V$, ind $F)$ is of type $\left(\mathrm{c}^{\prime}\right)$ and $l$ is odd; or

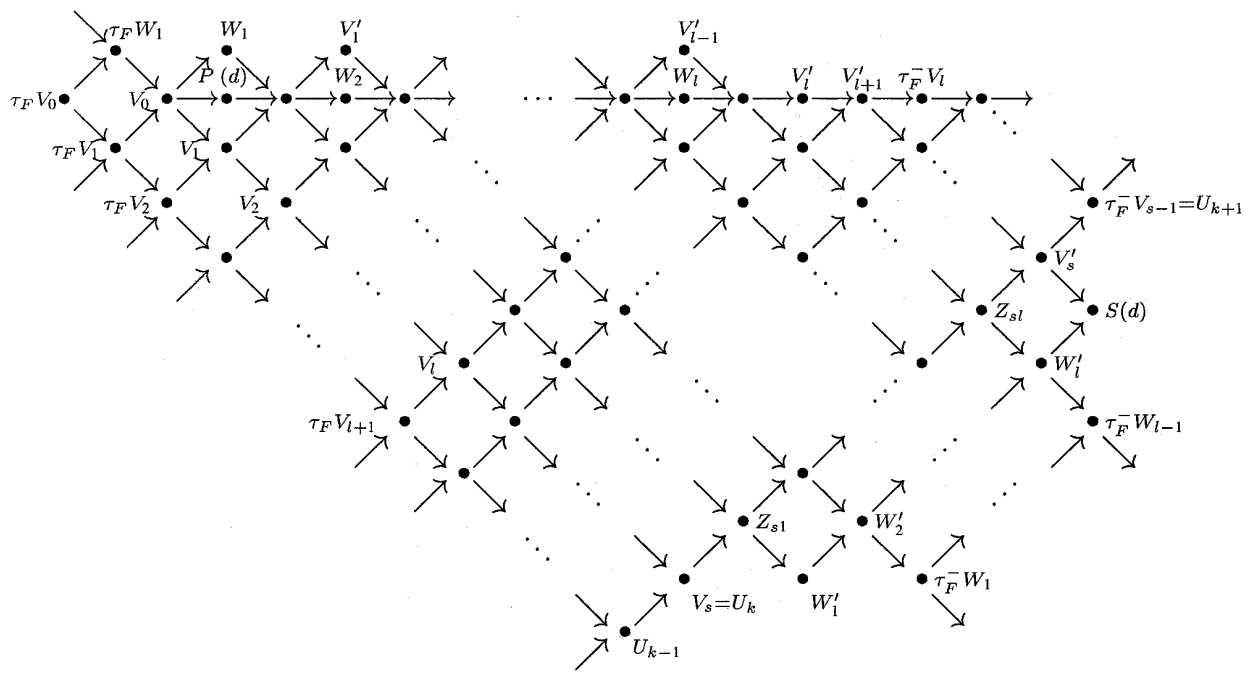

if $\operatorname{Hom}_{F}(V$, ind $F)$ is of type $\left(\mathrm{c}^{\prime}\right)$ and $l$ is even.

Observe that the sectional path $Y_{1}=U_{1} \rightarrow \cdots \rightarrow U_{r}$ is in this process extended to one of the sectional paths

$$
Y_{1} \rightarrow U_{1} \rightarrow \cdots \rightarrow U_{k} \rightarrow V_{s}^{\prime} \rightarrow U_{k+1} \rightarrow \cdots \rightarrow U_{r}
$$

or

$$
\begin{aligned}
Y_{1}= & U_{1} \rightarrow \cdots \rightarrow U_{k-1} \rightarrow U_{k} \rightarrow Z_{s 1} \rightarrow \cdots \rightarrow Z_{s l} \\
& \rightarrow V_{s}^{\prime} \rightarrow U_{k+1} \rightarrow \cdots \rightarrow U_{r} .
\end{aligned}
$$

Moreover, from the choice of $k$ and the shape of the inserted rectangle, we infer that none of $U_{1}, \ldots, U_{k}$ is the target of a sectional path with source being a direct summand of the radical of indecomposable projective module which is nonisomorphic to $P(d)$. If one of the modules $Z_{s 1}, \ldots, Z_{s l}, V_{s}, U_{k+1}, \ldots, U_{r}$ lies on a sectional path in $\mathcal{C}$ with source being direct predecessor of a projective module we repeat the above analysis. Since $\mathcal{C}$ admits only finitely many projective modules, we conclude that in fact $\mathcal{C}$ admits a finite sectional path

$$
\Theta: Y_{1} \rightarrow Y_{2} \rightarrow \cdots \rightarrow Y_{t}
$$

and $\operatorname{Hom}_{B}(M, \mathcal{C})$ is of the form (b). Observe also that after the extension of $A(x)$ to $A=A(x)[R(x)]$, any irreducible map $Y_{i} \rightarrow Z$ with $Z \not Y_{i+1}$ will belong, by Lemma 3.1 , to $\operatorname{rad}^{\infty}(\bmod A)$. In particular, any projective module which is successor of a module $Y_{i}, 1 \leqslant i \leqslant t$, lies on the path $\Theta$. 
Applying now [4] and [5] we conclude that, in all cases considered above, $M$ is the pivot of an admissible operation of type ( $\operatorname{ad} 1),(\operatorname{ad} 2)$, or $(\operatorname{ad} 3)$, and the component $\mathcal{C}^{\prime}$ of $\Gamma_{A}$ containing $P(x)$, is a standard multicoil. Moreover, it follows from Lemma 5.1 and the maximality of $x$ in $\mathcal{S}$, that any projective module which is successor of $P(x)$ in $\bmod A$ belongs to $\mathcal{C}^{\prime}$.

We shall describe the remaing components of $\Gamma_{A}$. First observe that, if $\mathcal{E}$ is a component in $\Gamma_{A(x)}$, such that $\operatorname{Hom}_{A(x)}(R(x), E)=0$ for all modules $E$ from $\mathcal{E}$, then $\mathcal{E}$ remains a full component in $\Gamma_{A}$, by [39, (2.5)]. Let $\mathcal{F}$ be a component of $\Gamma_{A}$ different from $\mathcal{C}^{\prime}$ but containing a module $Z$ with $\operatorname{Hom}_{A}(P(x), Z) \neq 0$. By [5, (4.1)], there exists a maximal tubular extension $\Omega$ of $C$ which is a convex subcategory of $A$ and $\mathcal{F}$ is a component of $\Gamma_{\Omega}$. It follows from Proposition 2.3 and our assumptions on $A$ that $\Omega$ is either (representation-infinite) tilted of Euclidean type with a complete slice in the preinjective component or tubular. In the first case, $\mathcal{F}$ is the unique preinjective component of $\Gamma_{\Omega}$. In the second case, $\mathcal{F}$ is, in the notations of Section 1, a component in

$$
\left(\bigvee_{\gamma \in \mathbb{Q}^{+}} \mathcal{T}_{\gamma}\right) \vee \mathcal{T}_{\infty} \vee \mathcal{I}_{\infty}
$$

We know that all components in $\Gamma_{\Omega}$ are standard and the component quiver $\Sigma_{\Omega}$ is directed. Hence, $\mathcal{F}$ is either a standard preinjective component or a standard tube without projective modules. Applying now our assumptions on $A(x)$, we conclude that $A$ is a multicoil algebra, all components in $\Gamma_{A}$ are standard, and $\Sigma_{A}$ is directed. This finishes the proof of Theorem 4.1 .

\section{References}

1. Assem, I. and Skowroński, A.: On some classes of simply connected algebras, Proc. London Math. Soc. 56 (1988) 417-450.

2. Assem, I. and Skowroński, A.: Minimal representation-infinite coil algebras, Manuscripta Math. 67 (1990) 305-331.

3. Assem, I. and Skowroński, A.: Indecomposable modules over multicoil algebras, Math. Scand. 71 (1992) 31-61.

4. Assem, I. and Skowroński, A.: Multicoil algebras, in: Representations of Algebras, CMS Conf. Proc. 14 (1993) 29-68.

5. Assem, I, Skowroński, A. and Tomé, B.: Coil enlargement of algebras, Tsukuba J. Math. 19 (1995) 453-479.

6. Auslander, M., Platzeck, M. I. and Reiten, I.: Coxeter functors without diagrams, Trans. Amer. Math. Soc. 250 (1979) 1-46.

7. Bautista, R., Gabriel, P., Roiter, A. V. and Salmeron, L.: Representation-finite algebras and multiplicative bases, Invent. Math. 81 (1985) 217-285.

8. Bautista, R., Larrion, F. and Salmeron, L.: On simply connected algebras, J. London Math. Soc. 27 (1983) 212-220.

9. Bongartz, K. On a result of Bautista and Smalø on cycles, Comm. Algebra 11 (1983) 2123-2124.

10. Bongartz, K.: Treue einfach zusammenhängende Algebren I, Comment. Math. Helv. 57 (1982) 282-330.

11. Bongartz, K.: Algebras and quadratic forms, J. London Math. Soc. 28 (1983) 461-469.

12. Bongartz, K.: Critical simply connected algebras, Manuscripta Math. 46 (1984) 117-136. 
13. Bongartz, K.: A criterion for finite representation type, Math. Annalen 269 (1984) 1-12.

14. Bongartz, K.: Indecomposables are standard, Comment. Math. Helv. 60 (1985) 400-410.

15. Bongartz, K. and Gabriel, P.: Covering spaces in representation theory, Invent. Math. 65 (1982) 331-378.

16. Bretscher, O. and Gabriel, P.: The standard form of a representation-finite algebra, Bull. Soc. Math. France 111 (1983) 21-40.

17. Crawley-Boevey, W. W.: On tame algebras and bocses, Proc. London. Math. Soc. 56 (1988) 451-483.

18. Dräxler, P.: Aufrichtige gerichtete Ausnahmealgebren, Bayreuter Math. Schriften 29 (1989).

19. Dräxler, P.: Sur les algèbres exceptionelles de Bongartz, C. R. Acad. Sci. Paris 311 (1990) 495-498.

20. Dräxler, P.: Completely separating algebras, J. Algebra 165 (1994) 550-565.

21. Drozd, Yu A.: Tame and wild matrix problems, in: Representation Theory II, Lecture Notes in Math. 832, Springer-Verlag (1980) 242-258.

22. Gabriel, P. and Roiter, A. V.: Representations of Finite-Dimensional Algebras, Algebra VIII, Encyclopedia Math. Sci. 73, Springer-Verlag, 1992.

23. Happel, D. and Vossieck, D.: Minimal algebras of infinite representation type with preprojective component, Manuscripta Math. 42 (1983) 221-243.

24. Kerner, O.: Tilting wild algebras, J. London Math. Soc. 39 (1989) 29-47.

25. Kerner, O. and Skowroński, A.: On module categories with nilpotent infinite radical, Compositio Math. 77 (1991) 313-333.

26. Lersch, M.: Minimal wilde Algebren, Diplomarbeit (Düsseldorf 1987).

27. Liu, S.: Semi-stable components of an Auslander-Reiten quiver, J. London Math. Soc. 47 (1993) 405-416.

28. Liu, S.: Infinite radicals in standard Auslander-Reiten components, J. Algebra 166 (1994) 245-254.

29. Nörenberg, R. and Skowroński, A.: Tame minimal non-polynomial growth simply connected algebras, Colloq. Math., in press.

30. Peng, L. G. and Xiao, J.: On the number of $D$ Tr-orbits containing directing modules, Proc. Amer. Math. Soc. 118 (1993) 753-756.

31. de la Peña, J. A.: On the representation type of one point extensions of tame concealed algebras, Manuscripta Math. 61 (1988) 183-194.

32. de la Peña, J. A.: Algebras with hypercritical Tits form, in: Topics in Algebra, Banach Center Publ. 26, Part 1 (PWN, Warsaw 1990) 353-369.

33. de la Peña, J. A.: Tame algebras with sincere directing modules, J. Algebra 161 (1993) 171-185.

34. de la Peña, J. A.: The families of two-parametric tame algebras with sincere directing modules, in: Representations of Algebras, CMS Conf. Proc. 14 (1993) 361-392.

35. de la Peña, J. A. and Skowroński, A.: Forbidden subalgebras of nonpolynomial growth tame simply connected algebras, Canadian J. Math. 48 (1996) 1018-1043.

36. de la Peña, J. A. and Tamé, B.: Iterated tubular algebras, J. Pure Appl. Algebra 64 (1990) 303-314.

37. Pogorzały, Z. and Skowroński, A.: On algebras whose indecomposable modules are multiplicityfree, Proc. London Math. Soc. 47 (1983) 463-479.

38. Ringel, C. M.: Finite dimensional hereditary algebras of wild representation type, Math. Z. 161 (1978) 236-255.

39. Ringel, C. M.: Tame algebras and integral quadratic forms, Lecture Notes in Math. 1099 (SpringerVerlag 1984).

40. Rogat, A. and Tesche, T.: The Gabriel quivers of the sincere simply connected algebras, Preprint 93-005, SFB 343 (Bielefeld).

41. Simson, D.: Linear representations of partially ordered sets and vector space categories, Algebra, Logic and Applications Series 4 (Gordon Breach Sci. Publ. 1992).

42. Simson, D.: Kronecker triangles and non-polynomial growth, C. R. Acad. Sci. Paris, t. 321, Serie I (1995) 33-38.

43. Skowroński, A.: Algebras of polynomial growth, in: Topics in Algebra, Banach Center Publications, 26, Part 1 (PWN, Warsaw 1990) 535-568. 
44. Skowroński, A.: Simply connected algebras and Hochschild cohomologies, in: Representations of Algebras, CMS Conf. Proc., 14 (1993) 431-447.

45. Skowroński, A.: Regular Auslander-Reiten components containing directing modules, Proc. Amer. Math. Soc. 120 (1994) 19-26.

46. Skowroński, A.: Cycles in module categories, in: Finite Dimensional Algebras and Related Topics, NATO ASI Series, Series C, Vol. 424, Kluwer Acad. Publ., Dortrecht 1994, pp. 309-345.

47. Skowroński, A.: Generalized standard Auslander-Reiten components, J. Math. Soc. Japan 46 (1994) 517-543.

48. Skowroński, A.: On semi-regular Auslander-Reiten components, Bull. Polish Acad. Sci., Mathematics 42 (1994) 157-163.

49. Skowroński, A.: Criteria for polynomial growth of algebras, Bull. Polish Acad. Sci., Mathematics 42 (1994) 173-183.

50. Skowroński, A.: Cycle-finite algebras, J. Pure Appl. Algebra 103 (1995) 105-116.

51. Skowroński, A.: Module categories over tame algebras, in: Representation Theory and Related Topics, Workshop Mexico 1994, CMS Conf. Proc. 19 (1996) 281-313.

52. Skowroński, A.: Tame algebras with strongly simply connected Galois coverings, Colloq. Math. 72 (1997) 335-351.

53. Skowroński, A. and Smalø, S. O.: Directing modules, J. Algebra 147 (1992) 137-146.

54. Skowroński, A. and Waschbüsch, J.: Representation-finite biserial algebras, J. Reine Angew. Math. 345 (1983) 172-181.

55. Unger, L.: The concealed algebras of the minimal wild, hereditary algebras, Bayreuter Math. Schriften 31 (1990) 145-154.

56. Wittman, J.: Verkleidete zahme and minimal wilde Algebren, Diplomarbeit (Bayreuth 1990). 